\title{
1 Avalanche criticality in individuals, fluid intelligence and working memory
}

2 Longzhou Xu (徐龙洲) ${ }^{a, 1}$, Lianchun Yu (俞连春) a, b, c, 1, *, Jianfeng Feng (冯建峰) d, e, f, *

3 a School of Physical Science and Technology, and Key Laboratory for Magnetism and

4 Magnetic Materials of MOE, Lanzhou University, Lanzhou, 730000, China

$5 \quad$ b Lanzhou Center for Theoretical Physics, Key Laboratory of Theoretical Physics of Gansu

6 Province, Lanzhou University, Lanzhou, Gansu 730000, China

$7 \quad{ }^{\mathrm{c}}$ The School of Nationalities' Educators, Qinghai Normal University, Xining, 810000, China

$8{ }^{d}$ Institute of Science and Technology for Brain Inspired Intelligence, Fudan University,

9 Shanghai, 200433, China

$10{ }^{\mathrm{e}}$ Department of Computer Science, University of Warwick, Coventry, CV4 7AL, UK

$11{ }^{\mathrm{f}}$ School of Mathematical Sciences, School of Life Science and the Collaborative Innovation

12 Center for Brain Science, Fudan University, Shanghai, 200433, PR China

13 * Corresponding authors: Lianchun Yu (yulch@lzu.edu.cn), Jianfeng Feng

14 (Jianfeng.Feng@warwick.ac.uk).

$15 \quad{ }^{1}$ These authors contributed equally to this work: Longzhou Xu, Lianchun Yu. 


\section{Abstract}

19 The critical brain hypothesis suggests that efficient neural computation can be

20 realized through dynamics of the brain characterized by scale-free avalanche activities. However, the relation between human cognitive performance and the avalanche criticality in large-scale brain networks remains unclear. In this study, we analyzed the mean synchronization and synchronization entropy of blood oxygenation level signals from resting-state fMRI. We found that the scale-free avalanche activity was associated with intermediate synchronization and maximal synchronization entropy. The complexity of functional connectivity, as well as structure-function coupling, is maximized by criticality, which is consistent with theoretical predictions. We observed order-disorder phase transitions in resting-state brain dynamics and found that there were longer times spent in the subcritical regime. These results support the hypothesis that large-scale brain networks lie in the vicinity of a critical point. Finally, we showed evidence that the neural dynamics of human participants with higher fluid intelligence and working memory scores are closer to criticality. We identified brain regions whose critical dynamics showed significant positive correlations with fluid intelligence performance, and found these regions were located in the prefrontal cortex and inferior parietal cortex, which are believed to be important nodes of brain networks underlying human intelligence. Our results reveal the role that avalanche criticality plays in cognitive performance, 
39 and provide a simple method to identify the critical point and map cortical states

40 on a spectrum of neural dynamics, ranging from subcriticality to supercriticality.

41 KEY WORDS: avalanche criticality, fluid intelligence, resting-state fMRI,

42 large-scale brain network, phase transition, working memory 


\section{Introduction}

The critical brain hypothesis states that the brain operates in close vicinity to a critical point that lies between order and disorder. This is characterized by a power-law form of the event size distribution (Cocchi, Gollo, Zalesky, \& Breakspear, 2017; Hesse \& Gross, 2014). This hypothesis is supported by a set of observations of power-law scaling in many different neural systems using various approaches (J. M. Beggs \& Plenz, 2003; Gal \& Marom, 2013; Meisel, Olbrich, Shriki, \& Achermann, 2013; Plenz, 2012; Shriki et al., 2013; Solovey, Miller, Ojemann, Magnasco, \& Cecchi, 2012; Enzo Tagliazucchi, Balenzuela, Fraiman, \& Chialvo, 2012). Arguments in favour of this hypothesis have been strengthened by advantages in information transmission, information storage, and dynamic range, in neural systems operating near criticality (Shew, Yang, Petermann, Roy, \& Plenz, 2009; Shew, Yang, Yu, Roy, \& Plenz, 2011; Yang, Shew, Roy, \& Plenz, 2012), with evidence arising in both theoretical and experimental work (Shew \& Plenz, 2012). Meanwhile, this hypothesis still faces challenges from several perspectives (J. Beggs \& Timme, 2012). For example, computational studies suggested that power laws may emerge from simple stochastic processes or noncritical neuronal systems (Touboul \& Destexhe, 2010), so power laws alone are prerequisite but not sufficient evidence for criticality. Meanwhile, it has been asked: "If the brain is critical, what is the phase transition (Fontenele et al., 2019)?" Indeed, the observation of power law 
avalanche activity along with a phase transition between order and disorder would be more persuasive for criticality. Furthermore, though previous studies have associated supercriticality with reduced consciousness (Meisel et al., 2013; Scott et al., 2014), near-critical dynamics with rest (Priesemann et al., 2014), and subcriticality with focused cognitive states (Fagerholm et al., 2015), there remains a gap between the specific brain state and efficient information processing endowed by criticality as predicted by theory (He, 2011). To fully understand the functional roles of critical and non-critical dynamics, more research is required to relate brain states and cognitive performance to neural dynamics that lie on a spectrum, ranging from subcriticality to supercriticality. To obtain a deeper understanding of this phenomenon, it is necessary to develop data analysis methods to represent this phase spectrum with high resolution and characterize the subsequent reorganization of brains with the transition in this spectrum (Fontenele et al., 2019).

With advances in brain imaging techniques such as functional magnetic resonance imaging (fMRI), the critical brain hypothesis has found roles in interpreting fundamental properties of large-scale brain networks in the context of structure-dynamics-function relationships (Karahanoğlu \& Van De Ville, 2017; Lee et al., 2019). For example, it has been shown that structural connections of brains are mostly reflected in functional networks, and this structure-function coupling is disrupted when brains move away from criticality 
86

during anesthesia (Enzo Tagliazucchi et al., 2016). Another application of criticality is to explain the dynamic basis of brain complexity (Popiel, 2020; E. Tagliazucchi \& Chialvo, 2013; Timme et al., 2016). In particular, functional connectivity (FC) complexity, which is an umbrella term describing the variability, diversity, or flexibility of functional connections in brain networks, has been associated with cognitive performance from many perspectives, such as high-order cognition, aging, and cognitive impairment in brain disorders (Ahmadlou, Adeli, Bajo, \& Adeli, 2014; Anokhin, Birbaumer, Lutzenberger, Nikolaev, \& Vogel, 1996; Omidvarnia, Zalesky, Van De Ville, Jackson, \& Pedersen, 2019; Smyser et al., 2016; B. Wang et al., 2017). Studies have suggested that the FC complexity may possibly be at its maximum at the critical point, while the FC capacity arises from special topological properties of the structural network, such as hierarchical modular organization (Song et al., 2019; R. Wang et al., 2019).

To validate these applications, both computer modeling methods and experimental data analysis methods were used. Computer modeling utilizes structural imaging data to model large-scale brain dynamics and functional networks (Deco, Jirsa, \& McIntosh, 2011; Nakagawa, Jirsa, Spiegler, McIntosh, \& Deco, 2013). However, there is still disagreement on which type of phase transition should be adopted for large-scale brain networks, e.g., first-order discontinuous vs. second-order continuous phase transitions and edge of chaos 
criticality vs. avalanche criticality (Kanders, Lorimer, \& Stoop, 2017; Scarpetta, Apicella, Minati, \& de Candia, 2018). Experimental studies usually take advantage of dynamic changes caused by interventions, such as deprived sleep, anesthesia, or brain diseases, to show deviations from criticality and subsequent reorganization of FC networks (Hobbs, Smith, \& Beggs, 2010; Meisel et al.,

Tagliazucchi et al., 2016). However, deviations caused by these interventions are usually unidirectional, either in the sub- or supercritical directions. Furthermore, it is not clear whether deviations from criticality caused by different intervention methods follow an identical phase transition trajectory.

117 Recent studies have proposed the concept of a "critical line" instead of a "critical point" and suggested that multiple phase transition trajectories may exist (Kanders, Lee, Hong, Nam, \& Stoop, 2020). Therefore, the successful retrieval of the phase transition trajectory from the large-scale brain networks will not only help to answer key questions regarding what the phase transition is if the brain is critical but also have important implications in brain functional imaging and large-scale brain modeling. In this study we applied a large number of criticality-related metrics to investigate the spatio-temporal dynamics of large scale brain networks as well as their variation in individuals, and explored associations with three different 

retrieved an inverted-U curve by mapping individuals' brains onto the phase plane between the mean synchronization (MS) and synchronization entropy (SE) of blood oxygenation level-dependent (BOLD) signals. We then performed the classic avalanche criticality analysis method on these data (J. M. Beggs \& Plenz, 2003; Enzo Tagliazucchi et al., 2012), and found that subjects who were closest to the critical point exhibited moderate mean and maximal variability in synchrony of BOLD signals (i.e., located around the tipping point of the inverted-U curve). This is consistent with previous findings that the neural systems operate neither at the synchronous nor the asynchronous ends of the spectrum, but rather near the critical point between them (Fontenele et al., 2019). We also showed that the large individual variation around the critical point could be the cause of the mismatch between group-level data analysis and theoretical prediction. However, this individual variation also gave us a chance to examine previous conjectures on criticality in large-scale brain networks. And we indeed found that both FC complexity and structure-function coupling were maximized around the criticality. We also utilized a sliding window approach to observe "instantaneous" phase transition occurring in individual brains. We 146 found that brains persisting in the subcritical regime exhibited longer dwell times than those in other regimes. Finally, we found the critical dynamics were associated with high scores in fluid intelligence and working memory tests, but 
not with crystallized intelligence scores. Additionally, the critical dynamics in the frontal cortex, superior parietal lobule, angular gyrus and supramarginal gyrus et al, which were believed as vital regions in the networks of Parieto-Frontal Integration Theory (P-FIT) for intelligence (Jung \& Haier, 2007), exhibited significant correlations with fluid intelligence performance.

\section{Material and methods}

\section{Data acquisition and preprocessing}

\section{fMRI data acquisition and preprocessing}

We used rfMRI data from the Human Connectome Project (HCP) 1200-subject release (Van Essen et al., 2013). Each subject underwent two sessions of rfMRI on separate days, each session with two separate $14 \min 24 \mathrm{~s}$ acquisitions generating 1200 volumes on a customized Siemens 3T Skyra scanner $(\mathrm{TR}=$ $720 \mathrm{~ms}, \mathrm{TE}=33 \mathrm{~ms}$, flip angle $=52^{\circ}$, voxel size $=2 \mathrm{~mm}$ isotropic , 72 slices

$$
\text { FOV }=208 \times 180 \mathrm{~mm} \text {, matrix }=104 \times 90 \mathrm{~mm}
$$

multiband accelaration factor $=8$, echo spacing $=0.58 \mathrm{~ms}$ ). The rfMRI data used for our analysis were processed according to the HCP minimal preprocessing pipeline (Glasser et al., 2016; Glasser et al., 2013) and denoising procedures. The denoising procedure pairs the independent component analysis with the FSL tool FIX to remove non-neural spatiotemporal components (Smith et al., 2015). And as a part of cleanup, HCP used 24 confound time series 
derived from the motion estimation (the 6 rigid-body parameter time series, their backwards-look temporal derivatives, plus all 12 resulting regressors squared). Note that the global component of the fMRI fluctuations measured during the resting state is tightly coupled with the underlying neural activity, and the use of global signal regression as a pre-processing step in resting-state fMRI analyses remains controversial and is not universally recommended (Liu, Nalci, \& Falahpour, 2017). Therefore, the global whole-brain signal was not removed in this work. We used the left-to-right acquisitions from the first resting-state dataset (i.e., resting state fMRI 1 FIX-denoised package).

The first 324 subjects in the dataset entered into our study, and we excluded 29 subjects for missing data. This left us with 295 subjects for further analysis, and 162 of them were females. All the participants were between the ages of 22-36, 58 were between the ages of 22-25, 130 were between the ages of 26-30, 104 were between the ages of $31-35$, and 3 were 36 years old.

For further analysis, the whole cortex was parcellated into 96 regions using the Harvard-Oxford atlas (Makris et al., 2006), and the details are provided at https://identifiers.org/neurovault.image:1699, and from each region the time series averaged across the voxels were extracted and Z-normalized to construct region of interest (ROI) signals (atlas96 signals). To test the results for different parcellation, we also used the Human Brainnetome atlas that comprised 246 regions (Fan et al., 2016), and Zalesky atlas that comprised 1024 regions 
(Zalesky et al., 2010), the resulting signals were termed as atlas 246 and atlas ${ }_{1024}$ signals, respectively.

\section{Diffusion tensor imaging (DTI) data acquisition and preprocessing}

The diffusion MRI images used in this study were also from the HCP 1200-subject release (Sotiropoulos et al., 2013). Briefly, the diffusion data were collected using a single-shot, single refocusing spin-echo, echo-planar imaging sequence $\left(\mathrm{TR}=5520 \mathrm{~ms}, \mathrm{TE}=89.5 \mathrm{~ms}\right.$, flip angle $=78^{\circ}, \mathrm{FOV}=210 \times$ $180 \mathrm{~mm}$, matrix $=168 \times 144 \mathrm{~mm}, \quad$ voxel size $=1.25 \mathrm{~mm}$ istropic slices $=111$, multiband acceleration factor $=3$, echo spacing $=0.78 \mathrm{~ms}$ ). Three gradient tables of 90 diffusion-weighted directions and six $b=0$ images, each were collected with right-to-left and left-to-right phase encoding polarities for each of the three diffusion weightings $\left(b=1000,2000\right.$, and $\left.3000 \mathrm{~s} / \mathrm{mm}^{2}\right)$. All diffusion data were preprocessed with the HCP diffusion pipeline updated with EDDY 5.0.10 (Sotiropoulos et al., 2013), and the details are provided at https://www.humanconnectome.org. In this study, from the 295 selected subjects, only 284 subjects were entered into our DTI data analysis because 11 of them were missing the corresponding DTI data.

\section{Cognition measures}

We examined associations between cognitive ability and critical dynamics conducted in our rfMRI analysis. Three relevant behavioral tasks were used as a 
210 measure of cognitive ability, including fluid intelligence, working memory, and

211 crystallized intelligence. The same 295 subjects were also entered into our

212 cognitive ability analysis, but for the fluid intelligence analysis, 5 subjects were

213 excluded due to missing their intelligence scores, or information about age and

214 education.

215 The fluid intelligence scores in the HCP data release were measured using the number of correct responses on form A of the Penn Matrix Reasoning Test

$($ PMAT, mean $=17.0034$, standard deviation $(\mathrm{SD})=4.9106$, range $=4-$

24), which had 24 items and 3 bonus items, using nonverbal visual geometric designs with pieces to assess reasoning abilities that can be administered in under 10 min (Barch et al., 2013; Hearne, Mattingley, \& Cocchi, 2016). The

PMAT (Bilker et al., 2012) is an abbreviated version of Raven's Standard

Progressive Matrices test (Wendelken, Nakhabenko, Donohue, Carter, \& Bunge, 2007), which comprises 60 items.

Crystallized intelligence was measured using the picture vocabulary test

(Picture vocabulary, mean $=116.8205, \mathrm{SD}=10.1977$, range=92.3914-153.0889) from the National Institutes of Health (NIH) toolbox (Barch et al., 2013; Hearne et al., 2016). This measure of receptive vocabulary was administered in a computer-adaptive testing (CAT) format. The participant was presented with

229 four pictures and heard an audio recording saying a word, and was instructed to 230 select the picture that most closely showed the meaning of the word. Because 
231 the test used a variable length CAT with a maximum of twenty-five items, some

232 participants had fewer items, and the presented words depended on the

233 participant's performance.

234 Working memory was assessed using the List Sorting Working Memory test

235 (List sorting, mean=111.2075, $\mathrm{SD}=12.0946$, range=84.63-144.50) from the $\mathrm{NIH}$

236 Toolbox (Barch et al., 2013), in which the participants were required to

237 sequence sets of visually and a small number of orally presented stimuli in size

238 order from smallest to biggest. Pictures of different foods and animals were

239 displayed with both a sound clip and a written test that names them, and 240 involved two different conditions. In the 1-list condition, participants ordered a 241 series of objects, either food or animals, but in the 2-list condition, participants

242 were presented with both animal and food lists and asked to order each list by

243 increasing size. The number of list items increased in subsequent trials, and the

244 task was discontinued after 2 consecutive incorrect trials.

\section{Data analysis methods}

Synchrony and variability in synchrony

247 We measured the mean and variability in synchronization with a previously

248 described approach (Meisel et al., 2013; Yang et al., 2012). First, we obtained

249 the phase trace $\theta_{j}(t)$ from the signal $F_{j}(t)$ using its Hilbert transform

$250 \quad H\left[F_{j}(t)\right]:$ 


$$
\theta_{j}(t)=\arctan \frac{H\left[F_{j}(t)\right]}{F_{j}(t)}
$$

252 Next, we calculated the Kuramoto order parameter as follows:

$$
r(t)=\frac{1}{u}\left|\sum_{j=1}^{u} e^{i \theta_{j}(t)}\right|
$$

254 in which $\mathrm{u}$ is the number of ROIs in global network analysis, or the number of voxels in a particular region in regional analysis. The Kuramoto order parameter

$256 r(t)$ was used as a time-dependent measure of phase synchrony of a system. The MS of a time period was calculated as

$$
\langle r\rangle=\frac{1}{V} \sum_{t=1}^{V} r(t),
$$
of the entire scan period with $V=1200$ time points. We derived the entropy of $r(t)$ as the measure of variability in synchronization (synchronization entropy, SE):

$$
H(r)=-\sum_{n=1}^{N} p_{n} \log _{2} p_{n},
$$

264 where $p_{n}$ is the probability that $r(t)$ falls into a bin between $\min (r(t)) \leq$ $b_{n}<r(t)<b_{n+1} \leq \max (r(t))$. In this study, we chose the number of

266 bins $N=30$, and the robustness of our results was also tested within an interval 267 between 5 and 100. 
270 point process by detecting the suprathreshold peak positions intermediate

271 between two above-threshold time points, as shown in the example in Fig. 1A.

By binning the binary sequences with appropriate time resolution (time bin), we

273 obtained a spatial pattern of active ROIs within consecutive time bins. An

274 avalanche was defined as a series of consecutively active bins, which were led

and followed by blank bins without activation. The size $\mathrm{S}$ and duration $\mathrm{T}$ of the

avalanches were then defined as the total number of activations and total

number of time bins during this avalanche, respectively (J. M. Beggs \& Plenz,

2003; Enzo Tagliazucchi et al., 2012).

If a system operates near a critical point, the size distribution $(P(S))$, duration distribution $(P(T))$, and average size for a given duration $(\langle S\rangle(T))$ should be

281 fitted into power laws:

$$
P(S) \sim S^{-\alpha}
$$

where $\alpha, \tau$, and $\gamma$ are critical exponents of the system (Friedman et al., 2012;

Sethna, Dahmen, \& Myers, 2001). Furthermore, the following scaling relation

was proposed as an important evaluation of the criticality (Fontenele et al., 2019;

288 Friedman et al., 2012), namely,

$$
\frac{\tau-1}{\alpha-1}=\gamma .
$$


In this study, we defined

$$
\delta=\left|\frac{\tau-1}{\alpha-1}-\gamma\right|
$$

292

to measure the distances of the systems from the critical point, so the smaller $\delta$ is, the closer the systems are to the critical point.

294 The scaling exponents governing the power-law distribution were estimated using the maximum likelihood estimator (MLE) (Clauset, Shalizi, \& Newman, 2009; Marshall et al., 2016). Briefly, the MLE procedure supposes that the empirical data sample is from a power-law function in the range $\left(x_{\min }, x_{\max }\right)$, 298 with probability density $\frac{1}{\sum_{x=x_{\min }}^{x_{\max }}\left(\frac{1}{x}\right)^{\alpha}}\left(\frac{1}{x}\right)^{\alpha}$ (Fontenele et al., 2019; Marshall et al., 2016). We estimated critical exponents $\alpha$ and $\tau$ by maximizing the likelihood function and via a lattice search algorithm (Marshall et al., 2016). We then used Clauset's goodness-of-fit test to quantify the plausibility of fits (Clauset et al., 2009; Deluca \& Corral, 2013; Marshall et al., 2016). We used a power-law model to produce data sets over the fit range and compared the Kolmogorov-Smirnov (KS) statistics between (1) the real data and the fit against (2) the model data and the fit. If the real data produced a KS-statistic that was less than the KS-statistic found for at least $10 \%$ of the power-law models (i.e., $\mathrm{p} \geq 0.1$ ), we accepted the data as being fit by the truncated power law because the fluctuations of the real data from the power law were similar in the KS sense to random fluctuations in a perfect power-law model. 
311 To assess the statistical significance of the avalanche analysis results and

312 MS-SE relationship, we generated comparable surrogate data and applied the analyses above to these data. Phase-shuffling is often used in hypothesis testing

314 for avalanche size distribution (Gireesh \& Plenz, 2008; Shriki et al., 2013).

315 Phase-shuffling disrupts temporal as well as spatial correlations in multichannel

316 time series.

317 Herein phase shuffling was done on the atlas96 signals. The phase randomization

318 procedures were as follows (Prichard \& Theiler, 1994): (1) the discrete Fourier

319 transformation was taken to of each subject; (2) rotating the phase at each

320 frequency by an independent random variable that was uniformly chosen in the range $[0,2 \pi]$. Crucially, the different time series were rotated by the different

322 phases to randomize the phase information; (3) the inverse discrete Fourier transformation was applied to these time series to yield surrogate data.

\section{Branching parameter}

325 The branching parameter $\sigma$, which is defined as the average number of subsequent events that a single preceding event in an avalanche triggers, is a convenient measure to identify criticality (J. M. Beggs \& Plenz, 2003). In theory, the system is critical for $\sigma=1$ and sub- (super) critical for $\sigma<1(\sigma>1)$. In this study, $\sigma$ was calculated as 


$$
\sigma=\frac{1}{N} \sum_{i=1}^{N} \frac{n_{i}^{*}}{n_{i}}
$$

331 where $n_{i}$ is the number of ancestors, $n_{i}{ }^{*}$ is the number of descendants in the next time bin, and $N$ is the total number of time bins with activations.

A nonparametric measure, $\kappa$, for neuronal avalanches was introduced by Shew and his colleagues (Shew et al., 2009). It quantifies the difference between an experimental cumulative density function (CDF) of the avalanche size, $F\left(\beta_{k}\right)$, and the theoretical reference $\mathrm{CDF}, F^{N A}\left(\beta_{k}\right)$, which is a power-law function with theoretical expected exponent $\alpha=1.5$ :

$$
\kappa=1+\frac{1}{m} \sum_{k=1}^{m}\left(F^{N A}\left(\beta_{k}\right)-F\left(\beta_{k}\right)\right),
$$

340 where $\beta_{k}$ are avalanche sizes logarithmically spaced between the minimum and maximum observed avalanche sizes, and $m$ is the number of histogram

342 bins. The unit value of $\kappa$ is characteristic of the system in a critical state, 343 whereas values below and above 1 suggest sub- and supercritical states, 344 respectively.

\section{Functional and structure connectivity matrix}

346 We constructed an FC matrix from atlas96 signals by computing the Pearson 347 correlation $C_{i j}$ between ROI $i$ and ROI $j$, and the mean FC strength $\langle F C\rangle$ was obtained by 


$$
\langle F C\rangle=\left\langle\left|C_{i j}\right|\right\rangle,
$$

350 where $|\cdot|$ means the absolute value.

351 The structure connectivity (SC) matrix was constructed using DSI Studio

352 (http://dsi-studio.labsolver.org) from DTI data. The DTI data were 353 reconstructed in the Montreal Neurological Institute (MNI) space using 354 q-space diffeomorphic reconstruction (F.-C. Yeh \& Tseng, 2011) to obtain the spin distribution function (F. Yeh, Wedeen, \& Tseng, 2010). A diffusion sampling length ratio of 1.25 was used. The restricted diffusion was quantified using restricted diffusion imaging (F.-C. Yeh, Liu, Hitchens, \& Wu, 2017), and a deterministic fiber tracking algorithm (F.-C. Yeh, Verstynen, Wang, Fernández-Miranda, \& Tseng, 2013) was used to obtain one million fibers with whole-brain seeding. The angular threshold was randomly selected from 15

361 degrees to 90 degrees. The step size was randomly selected from 0.1 voxels to 3 voxels. The anisotropy threshold was automatically determined by DSI Studio. The fiber trajectories were smoothed by averaging the propagation 364 direction with a percentage of the previous direction. The percentage was 365 randomly selected from $0 \%$ to $95 \%$. Tracks with a length shorter than 5 or 366 longer than $300 \mathrm{~mm}$ were discarded. The SC matrix was calculated by using 367 the count of the connecting tracks using 96-region Harvard-Oxford atlas. 
The FC entropy $H(F C)$ is calculated by

$$
H(F C)=\sum_{i}-p_{i} \log _{2}\left(p_{i}\right)
$$

371 where $p_{i}$ is the probability distribution of $\left|C_{i j}\right|$, i.e., $\sum_{i} p_{i}=1$ (Yao et al.,

372 2013). In the calculation, the probability distribution was obtained by 373 discretizing the interval $(0,1)$ into 30 bins.

374

\section{Functional connectivity diversity}

The functional diversity $(D(F C))$ of the FC matrix is measured by the similarity of the distribution to the uniform distribution (R. Wang et al., 2019):

$$
D(F C)=1-\frac{1}{N_{M}} \sum_{i=1}^{M}\left|p_{i}-\frac{1}{M}\right|,
$$

where $N_{M}=2 \frac{M-1}{M}$ is a normalization factor, $D(F C)$ is in the range $[0,1]$, and $p_{i}$ is the probability distribution of $\left|C_{i j}\right|$, which was obtained by discretizing the interval $(0,1)$ into $\mathrm{M}$ bins $(M=30$ in this work). For completely asynchronous or synchronized states, the correlation values fall into one bin at 0 or 1 , where $D(F C)=0$ reflects the simple dynamic interaction pattern. In an extreme case where all types of FC equivalently exist, $p_{i}$ would ideally follow a uniform distribution (i.e., probability in each bin $=\frac{1}{M}$ ) and $D(F C)=1$.

\section{Functional connectivity flexibility}

To obtain the flexibility of FC in the whole brain, we utilized the sliding 
window method to calculate connectivity number entropy ( $C N E$ ) for each region (Lei et al., 2020; Song et al., 2019). A non-overlapping sliding window method was applied to the atlas96 signals. The choice of window size must be sufficient to yield a stable Pearson's correlation coefficient within each window, yet small enough to reveal the temporal-dependent variation in FC (Lei et al., 2020; Sakoğlu et al., 2010). We chose a window size in the range of 20-30, corresponding to the number of windows $\left(n_{\text {win }}\right)$ in the range of $40-60$.

Within each time window, we first acquired the FC matrix via their time series in this window. Then, the binary network matrix was obtained by binarizing the FC matrix with a threshold $T H R_{F C}$. Subsequently, we calculated the number of regions connected to a particular region $k(k=1,2, \ldots 96)$ in each time window. Therefore, we could obtain $p_{i}$, the probability for a particular connection number occurring, where $i$ indicated the $i$-th connection number among all possible connection numbers. Then, for the region $k, C N E_{k}$ is a complexity measure (i.e., Shannon entropy) for the disorder in the connection numbers over time:

$$
C N E_{k}=-\sum_{i=1}^{95} p_{i} \log _{2} p_{i},
$$

where the summation index runs from 1 to the number of all possible connection numbers.

406 For each subject, the $C N E$ at the whole-brain level was obtained by simply 
407

408

409

averaging the regional $C N E_{k}$ values over 96 regions:

$$
C N E=\frac{1}{96} \sum_{k=1}^{96} C N E_{k}
$$

\section{Similarity between functional and structural networks}

To measure the similarity between functional and structural connection networks, the FC matrices $C_{i j}$ were thresholded by $T H R_{F C}$ to yield binary adjacency matrices $A_{i j}$ such that $A_{i j}=1$ if $C_{i j} \geq T H R_{F C}$, and $A_{i j}=0$ otherwise. The parameter $T H R_{F C}$ was chosen to fix link density $\rho_{F C}$, which was defined as the ratio of the connections in the network $\left(\sum_{i>j} A_{i j}\right)$ to the total possible number of connections. It is important to fix the link density when comparing networks, as otherwise, differences could arise because the average of the respective $C_{i j}$ are different (and therefore the number of nonzero entries in $A_{i j}$ ) but not because connections are topologically reorganized across conditions (Enzo Tagliazucchi et al., 2016).

The binary FC networks for each subject were compared with the group-aggregated binary SC network but not with the individual's SC network to avoid fluctuations in individual SC networks. First, the binary adjacency matrices $B_{i j}$ of SC matrices were obtained for each subject such that $B_{i j}=1$ if there were tracked fiber links; otherwise, $B_{i j}=0$. Then, the binary adjacency structural connection matrices were summed up and again thresholded by a thresholding value $T H R_{S C}$ to yield a group-aggregated binary SC network. In 
427 this way, high $T H R_{S C}$ values would exclude connections that were shared by

428 fewer subjects but preserve connections that were common in most subjects.

429 To estimate the similarity between the binary FC network of each subject and

430 the group-aggregated binary SC network, we computed the Pearson correlation

$431 \quad R(F C-S C)$ and Hamming distance $H D(F C-S C)$ between these two 432 networks (Enzo Tagliazucchi et al., 2016). Specifically, the Hamming distance 433 is defined as the number of symbol substitutions (in this case 0 or 1 ) needed to 434 transform one sequence into another and vice versa, and in this case, it is equal 435 to the number of connections that must be rewired to turn the functional 436 network connection into the structural network connection.

\section{Dynamic analysis of phase transitions}

438 We used the sliding window approach to capture the time-dependent changes in measures used in this study. In the calculation, for the atlas96 signals, the length

440 of the sliding window was set to $V=200$ (volumes), and the sliding step was 441 set to $\Delta n=10$ (volumes). In each window, we calculated the corresponding 442 dynamic measures, including dynamic MS $\langle r\rangle_{n}$, dynamic SE $H(r)_{n}$, and 443 dynamic FC matrix $\left(C_{i j}\right)_{n}$. From the dynamic FC matrix $\left(C_{i j}\right)_{n}$, we further 444 obtained dynamic FC entropy $H(F C)_{n}$, FC diversity $D(F C)_{n}$, Pearson 445 correlation between $\mathrm{FC}$ and $\mathrm{SC} \mathrm{R}(\mathrm{FC}-\mathrm{SC})_{\mathrm{n}}$, and Hamming distance 446 $H D(F C-S C)_{n}$ 
448 The rfMRI data, DTI data and cognitive data are available from the Human

449 Connectome Project at humanconnectome.org, WU-Minn Consortium. The

450 WU-Minn HCP Consortium obtained full informed consent from all participants,

451 and research procedures and ethical guidelines were followed in accordance

452 with the Washington University Institutional Review Boards (IRB \#201204036;

453 Title: 'Mapping the Human Connectome: Structure, Function, and Heritability').

454 MATLAB (https://www.mathworks.com/) and SPSS

455 (https://www.ibm.com/analytics/spss-statistics-software) were used to conduct 456 the experiment's reported in this study. The datasets supporting this article and 457 the codes required to reproduce them can be found at 458 https://github.com/longzhou-xu/data_and code_sort.git . 


\section{Results}
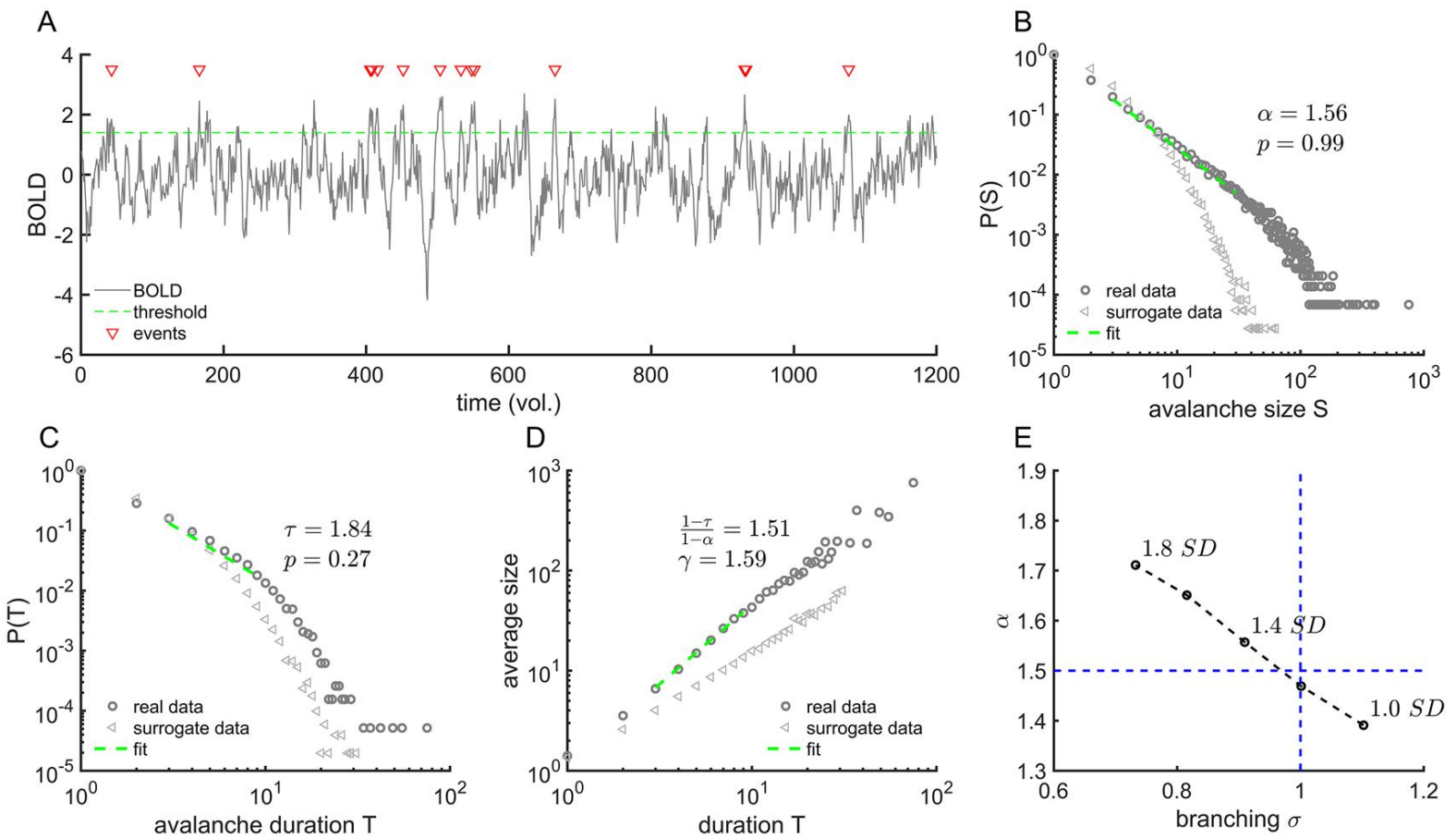

Figure 1. Avalanche statistics obtained from group-level analysis. A. Example of a point

process (red triangles) extracted from one normalized ROI BOLD signal. B. The probability

distributions of group-aggregated avalanche sizes for the threshold 1.4 SD and the time bin

width of 1 volume (vol.) in the fMRI data. The distributions are well approximated by power law with an exponent of $\alpha=1.56$ with Clauset's test $p=0.99$, corresponding to $\kappa=$ 0.9912 (histogram bins $=40$ ) and branching parameter $\sigma=0.9097$. C. The distribution of avalanche durations can be fitted well by a power law with an exponent of $\tau=1.84$ under the condition described in $\mathbf{B}$, with Clauset's test $p=0.3$. D. There is a relation between the 470 sizes and duration of the avalanches with a positive index $\gamma=1.59$, which is close to $\frac{1-\tau}{1-\alpha}=$ 
472 branching ratio and power-law scaling exponents $\alpha$ of avalanche sizes for different 473 thresholds used to define the point process.

474 For the 295 available subjects, we first investigated the power-law distribution 475 of avalanche size at the population level. Here, we defined the activation as the 476 time point when the BOLD signals reached their peak value, while the signals one step before and after this time point were above the chosen threshold (Fig. 1A). After preprocessing, the atlas 96 signals were converted into point processes 479 in which each time point represented an activation. We then calculated the 480 avalanche size distribution $P(S) \sim S^{-\alpha} \quad$ (Fig. 1B), as well as the avalanche 481 duration distribution $P(T) \sim T^{-\tau}$ (Fig. 1C). First, from the estimated $\alpha$ and $\tau$ values, we tested whether the relationship between the scaling exponents holds for different thresholds of ROI signals (Fontenele et al., 2019; Friedman et al., 2012). We found that the closest matching occurred when the chosen threshold was around 1.4 SD (Fig. 1D). Second, the power-law distribution of avalanche sizes with a slope of $\alpha=1.5$ could be predicted by theory for a critical branching process with branching parameter $\sigma=1$ (Harris, 1964; Zapperi, Lauritsen, \& Stanley, 1995). However, we found that the threshold of 1.4 SD yielded $\sigma=0.91$ and $\alpha=1.56$ (Fig. 1E), which did not match well with the theoretical prediction. We ran the same analysis on both atlas 246 signals (Fig.

S1a) and atlas ${ }_{1024}$ signals (Fig. S1b) to find the mismatch still exists (Fig. 

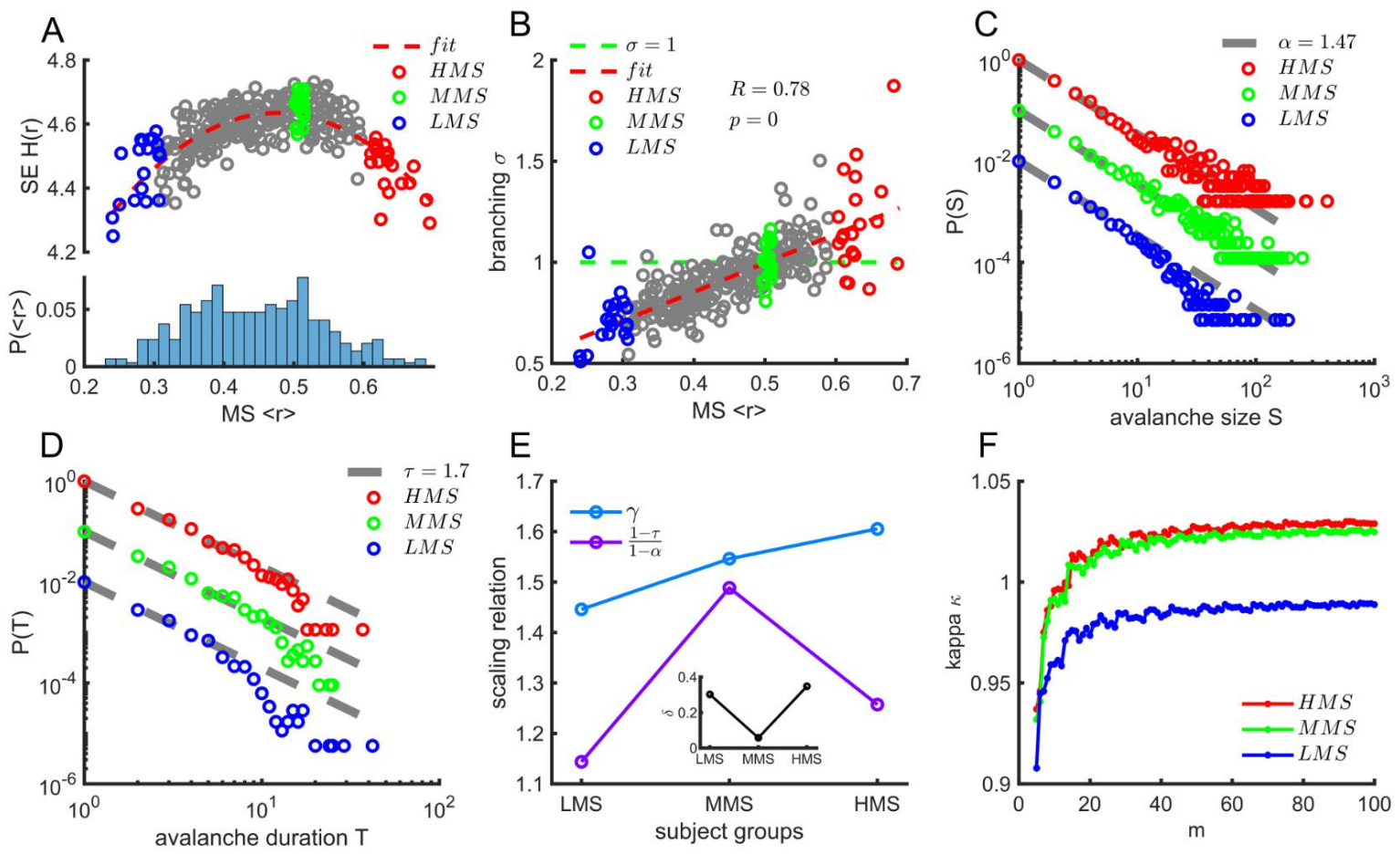

495 Top panel: The inverted-U trajectory of the MS $\langle r\rangle$ vs. SE $H(r)$. The red dash line 496 represents the quadratic fit of the data $\left(F=188.758, p<0.001\right.$, adjusted $\left.R^{2}=0.561\right)$. Bottom panel: The frequency count for the distribution of MS $\langle r\rangle$. B. The branching parameters $\sigma$ vs. $\langle r\rangle$ for each subject. The green dashed line indicates $\sigma=1$. The Pearson correlation value $R$ and the $p$ value are shown in the figure. The red dashed line represents the linear regression. For further analysis, we selected three representative groups of subjects according to their synchronization level: namely, LMS group $(\langle r\rangle=0.2824 \pm 0.0219$, blue open circles in A and B), MMS group $(\langle r\rangle=0.5041 \pm 0.0042$, green open circles in A and B) and

HMS group $(\langle r\rangle=0.6304 \pm 0.0246$, red open circles in A and B). C. Avalanche size distributions for the LMS group, MMS group, and HMS group. To show the difference between these groups, we used gray lines with $\alpha=1.47$ to guide the eyes. The 
corresponding group-aggregated branching parameters are $\sigma_{L M S}=0.7237$ for the LMS group, $\sigma_{M M S}=1.0123$ for the MMS group, and $\sigma_{H M S}=1.2023$ for the HMS group. D. groups, we used gray lines with $\tau=1.7$ to guide the eyes. E. Scaling relations for the three

510 groups. The blue line and purple line correspond to $\gamma$ and $\frac{\tau-1}{\alpha-1}$, respectively. In the inset, $511 \delta=\left|\gamma-\frac{\tau-1}{\alpha-1}\right|$ indicates the distance to the critical point. F. The dependence of $\kappa$ on the 512 numbers $m$ of histogram bins for the three groups.

513 As the above close check of hallmarks of criticality did not agree with each 514 other well, we moved forward to investigate whether this mismatch could be a result of intersubject variability. We calculated both the MS and SE (see Synchrony and variability in synchrony) using the atlas 96 signals for each of the 295 subjects and characterized the brain states of each subject with points in the MS vs. SE phase plane, as seen in the top panel of Fig. 2A. We found that the value of MS from these subjects extended from 0.2 to 0.7 , and the distribution of subjects was not even but exhibited a greater tendency to the low MS range (Fig. 2A, bottom panel). This result suggested that even in the resting state, there is significant variability among the subjects' brain states. It is clearly seen that these state points formed an inverted-U trajectory in the phase plane. The SE exhibited a maximum at the moderate value of MS, which implied the existence of a state with dynamic richness between order and disorder. We found the inverted-U curves and the calculation of SE were robust against 
527 different parcellation (Fig. S2). We also performed a phase randomization method on the fMRI data and found that this inverted-U curve disappeared in

529 the randomized surrogate datasets $($ size $=500$, identified by visual inspection;

530 examples can be seen in Fig. S3). Therefore, we argued that this inverted-U curve reflected a special spatiotemporal structure of brain dynamics that did not exist in randomized data.

As expected, with the increasing of MS, the spatiotemporal activation pattern defined before exhibited transitions from random states to ordered states (Fig.

S4). We then calculated the branching parameter $\sigma$ for each subject. We found that with increasing MS, the branching parameter increased from less than 1 to higher than 1, crossing 1 at a moderate value of MS (Fig. 2B, and Fig. S5 for different parcellation).

Furthermore, we selected three groups from the above subjects: the low mean synchronization group (LMS group; the 20 most left subjects in Fig. 2A with an

MS value of $\langle r\rangle=0.2824 \pm 0.0219$ ), the moderate mean synchronization group (MMS group; the 20 subjects located near the peak of curve in Fig. 2A with an MS value of $\langle r\rangle=0.5041 \pm 0.0042$ ), and the high mean synchronization group (HMS group; the 20 most right subjects in Fig. 2A with an MS value of $\langle r\rangle=0.6304 \pm 0.0246$ ). For each group, we performed 546 avalanche distribution analysis to identify which group was closest to the 547 critical point (Fig. 2C-F). After obtaining scaling exponents $\alpha$ and $\tau$ for each 
group with a threshold of 1.4 SD (Fig. 2C-D), the scaling relationship showed

549 the best match for the MMS group (Fig. 2E), and more detailed analysis results

550 can be found in Fig. S6 in the Supplementary Materials.

551 Previous study showed that the truncations of power-law fit have a dramatic

552 impact on power-law exponents, particularly on the ratio $\frac{1-\tau}{1-\alpha}$, while $\gamma$ barely 553 changes (Destexhe \& Touboul, 2020). To test the robustness of our results, we performed analysis in Fig 2c-e with different fitting windows for avalanche size

$\mathrm{S}\left(S_{\min } \in[1,10]\right.$, and $\left.S_{\max } \in[30,60]\right)$ and avalanche duration $\mathrm{T}\left(T_{\min } \in\right.$

$[1,5]$, and $\left.T_{\max } \in[9,20]\right)$. We found that the ratio of the number of fittings

that met the critical criterion $(|(1-\tau) /(1-\alpha)-\gamma|<0.1$ and Clauset's

goodness-of-fit test $p>0.1$ ) to all power-law-fit samples is highest for MMS

group ( $\varphi=0.0346$ for LMS, $\varphi=0.1103$ for MMS, $\varphi=0.0266$ for MMS).

We also calculated $\kappa$, an often-used parameter that could distinguish the difference between data and the theoretically suggested power-law distribution

(Fagerholm et al., 2015; Palva et al., 2013; Poil, Hardstone, Mansvelder, \&

Linkenkaer-Hansen, 2012; Shew et al., 2009; Shew et al., 2011). As shown in

Fig. $2 \mathrm{~F}$, as the discrete bin number $m$ increases, the $\kappa$ values become stable.

The stabilized $\kappa$ is smaller than 1 for the LMS group but larger than 1 for the

HMS and MMS groups. The $\kappa$ value for MMS group was closest to 1 .

Therefore, the above results suggested that subjects' brains with moderate MS and maximal SE are poised closest to the critical point, supported by consistent 


\section{The complexity in the FC network is maximized by criticality}
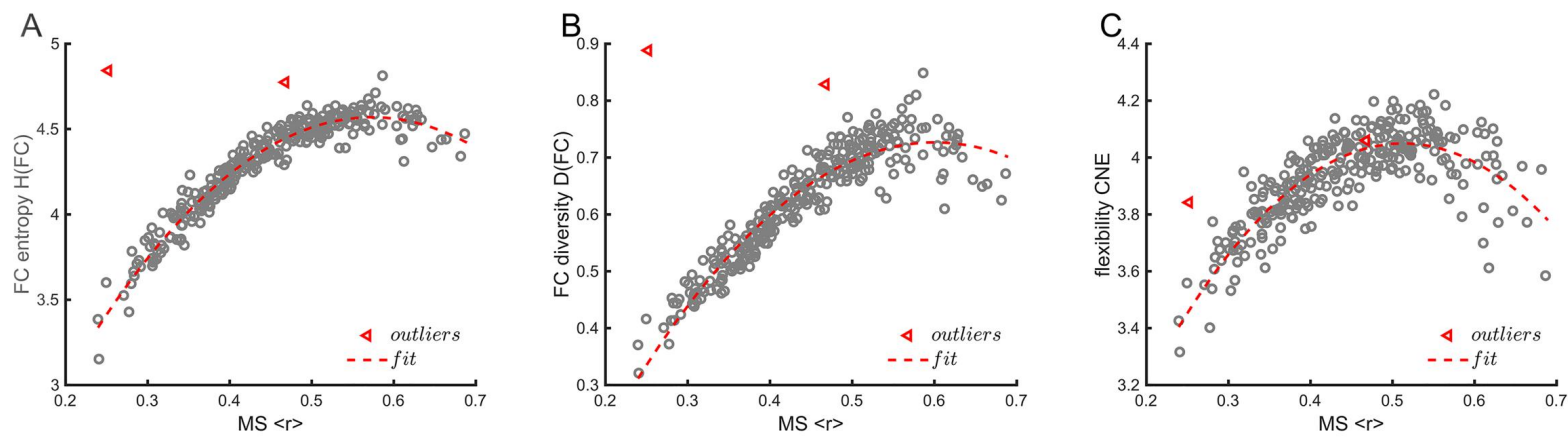

Figure 3. Dependence of complexity in the FC network on the MS of BOLD signals. A.

FC entropy $H(F C)$ as a function of MS $\langle r\rangle$. Red dashed line: quadratic fitting ( $F=$

Red dashed line: quadratic fitting $\left(F=1226.057, p<0.001\right.$, adjusted $\left.R^{2}=0.894\right)$. $p<0.001$, adjusted $\left.R^{2}=0.715\right)$. The red open triangles represent participants with outliers in the quadratic fittings in A-B.

Since the disorder-order phase transition could be observed, we investigated how this phase transition could impact the organization of FC networks. For convenience, we used MS to indicate this transition. We assessed how the variousness in FC strength changes as the brain undergoes a phase transition 
586 from the sub- to supercritical states. We used FC entropy and FC diversity as measures of variousness in FC strength in the brain networks. FC entropy is a direct measure of Shannon entropy from the probability distribution of FC strength obtained from the FC matrix, whereas FC diversity measures the similarity between the distribution of real FC matrix elements and uniform distribution. In previous studies, the former had been associated with healthy aging (Yao et al., 2013), and the latter is predicted to be maximized at the critical point by a computer model with Ginzburg-Landau equations (R. Wang et al., 2019). We found that both FC entropy (Fig. 3A) and FC diversity (Fig. 3B) peaked at the moderate value of MS; however, the peak position for these two measures was more rightward than that of SE.

The flexibility in dynamic $\mathrm{FC}$ reflects the extent of abundant connection patterns among regions and how frequent switching may occur between different patterns. In this work, we adopted the connection number entropy $600(C N E)$ as a measure of flexibility in FC networks. Our previous study showed 601 that this measure was maximized at the critical point in a large-scale brain network model that combined DTI structural data and excitable cellular automaton (Song et al., 2019), and this measure could be reduced in the brains 604 of patients with moyamoya disease (Lei et al., 2020). In this study, we found 605 that the flexibility in FC was maximized with a moderate value of MS (Fig. 3C). 606 The maximization was robust in a wide range of $T H R_{F C}$ thresholds and sliding 

peak position for FC flexibility was nearer to the critical point.

610 FC entropy, diversity, and flexibility are often used in rfMRI studies to measure

611 the complexity in the structure and dynamic reconfiguration of FC networks.

612 Here, the study suggested that the complexity in FC networks is maximized by 613 criticality.

\section{The maximized structure-function coupling around the critical point}

A

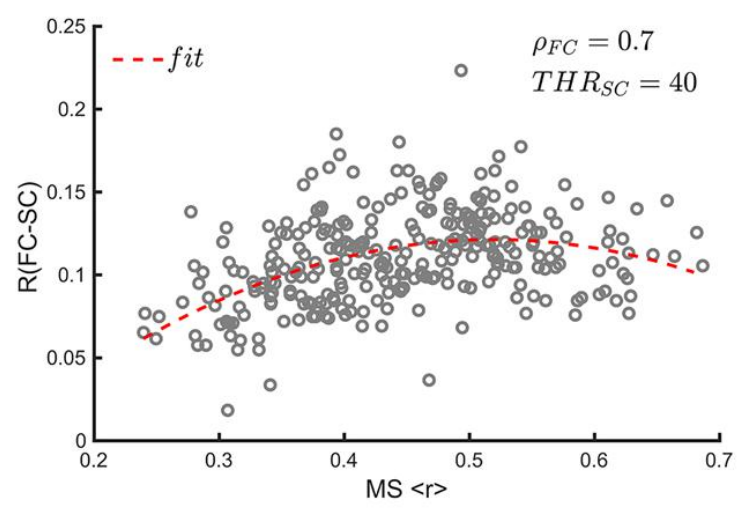

C

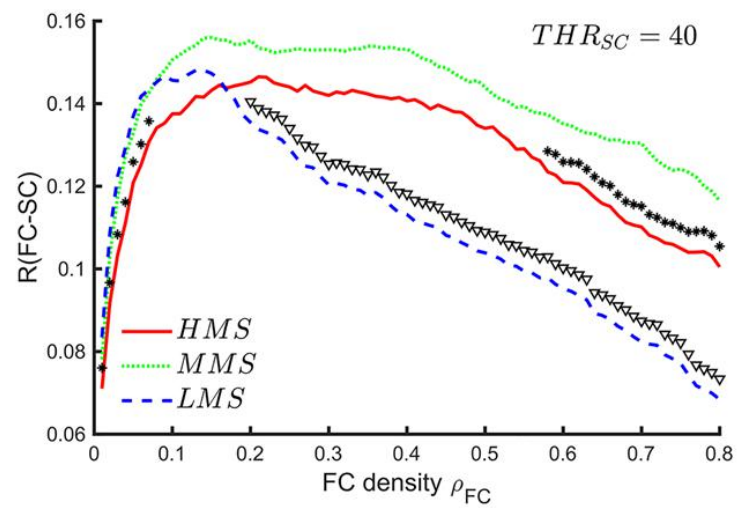

B

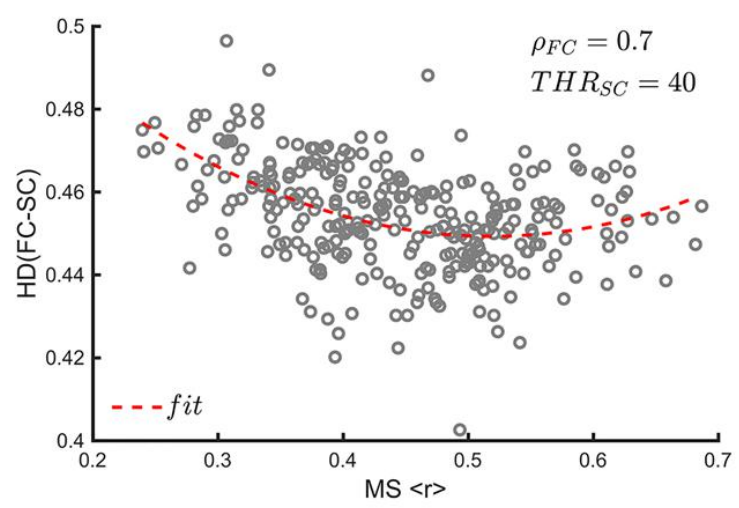

$\mathrm{D}$

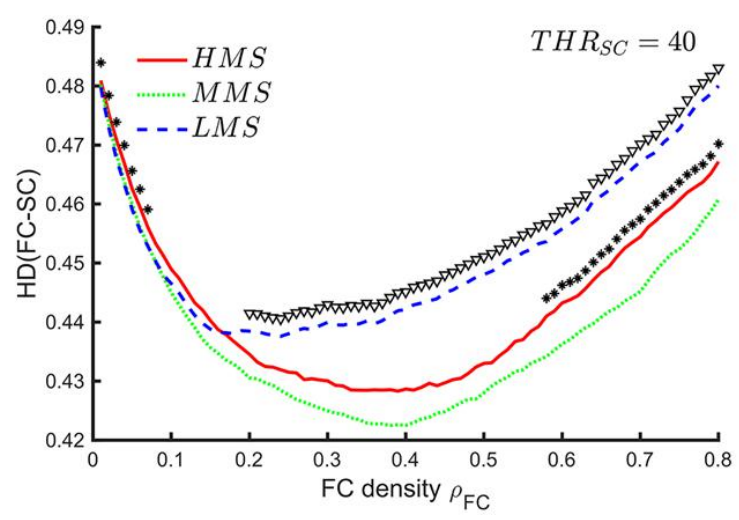

Figure 4. The dependence of structure-function coupling on the MS of brain networks.

617 A. Pearson correlation between anatomical and functional networks as a function of MS $\langle r\rangle$. 
618 The link density in the FC network $\rho_{F C}=0.7$ and threshold in the group-aggregated SC 619 network $T H R_{S C}=40$ (corresponding to $\rho_{S C}=0.4836$ ) are shown in the figure. Red dashed 620 line: quadratic fitting $\left(F=36.997, p<0.001\right.$, adjusted $\left.R^{2}=0.197\right)$, which is better than 621 linear fitting ( $F=39.346, p<0.001$, adjusted $R^{2}=0.115$ ). B. Hamming distance $H D(F C-S C)$ between anatomical and functional networks as a function of MS $\langle r\rangle$. Red dashed line: quadratic fitting $\left(F=36.997, p<0.001\right.$, adjusted $\left.R^{2}=0.197\right)$, which is better than linear fitting $\left(F=39.346, p<0.001\right.$, adjusted $\left.R^{2}=0.115\right)$. C. The Pearson correlation between anatomical and functional networks as a function of FC density $\rho_{F C}$ $\left(T H R_{S C}=40\right.$ corresponding to $\rho_{S C}=0.4826$ ) for the HMS, MMS, and LMS groups. D. The Hamming distance between anatomical and functional networks as a function of FC density $\rho_{F C}$ $\left(T H R_{S C}=40\right.$ corresponding to $\left.\rho_{S C}=0.4826\right)$ for the HMS, MMS, and LMS groups. In C-D, the stars indicate significant differences between the HMS and MMS groups (two-tails two-sample t-test, $p<0.05$, uncorrected); the open triangles indicate significant differences between the LMS and MMS groups (two-tails two-sample t-test, $p<0.05$, uncorrected).

From the obtained FC matrix and SC matrix for each subject, we constructed the FC networks for each subject and a group-aggregated SC network (see Similarity between functional and structural networks). We used $T H R_{F C}$ and $T H R_{S C}$ to control the link density in the FC networks and the group-aggregated SC network, respectively. We measured the similarity between the FC network and group-aggregated SC network with Pearson correlation and Hamming 
each subject with a link density of 0.7 in the FC networks. The similarity

640 between the FC and SC was maximal for subjects with moderate synchrony, as

641 the Pearson correlation was maximized (Fig. 4A), while the Hamming distance

642 was minimized (Fig. 4B) for these subjects. This maximization of similarity between the FC and SC could be observed in a wide range of link densities in

644 the FC and SC networks. To further consolidate the above results, we measured 645 the similarity between the FC and SC for the three groups (LMS MMS and HMS) defined above as a function of the FC network link density. Fig. 4C-D show that as the FC network link density increased, the correlation coefficient 648 between the FC and SC matrices first increased and then decreased, and 649 consistently, the Hamming distance exhibited the opposite tendency. When the 650 FC link density was large, the MMS group showed a significantly higher correlation and a lower Hamming distance between the FC and SC networks 652 than the other two groups. Similarly, by varying $T H R_{S C}$, we found that the maximized similarity in the $\mathrm{FC}$ and $\mathrm{SC}$ at the critical point was robust in the wide range of link densities in the SC network (Figs. S8, S9 and S10).

We noticed that for a large link density of the FC network, the dependence of similarity on link density monotonically decreased (Fig. 4C-D; Fig. S8a-b).

657 Since lower link density conserved only stronger links in FC networks, we 658 deduced that structural connections were mostly reflected in the strong 659 functional connections. Meanwhile, the similarity also decreased as the 


\section{The dynamic phase transition in individual subjects' brains}
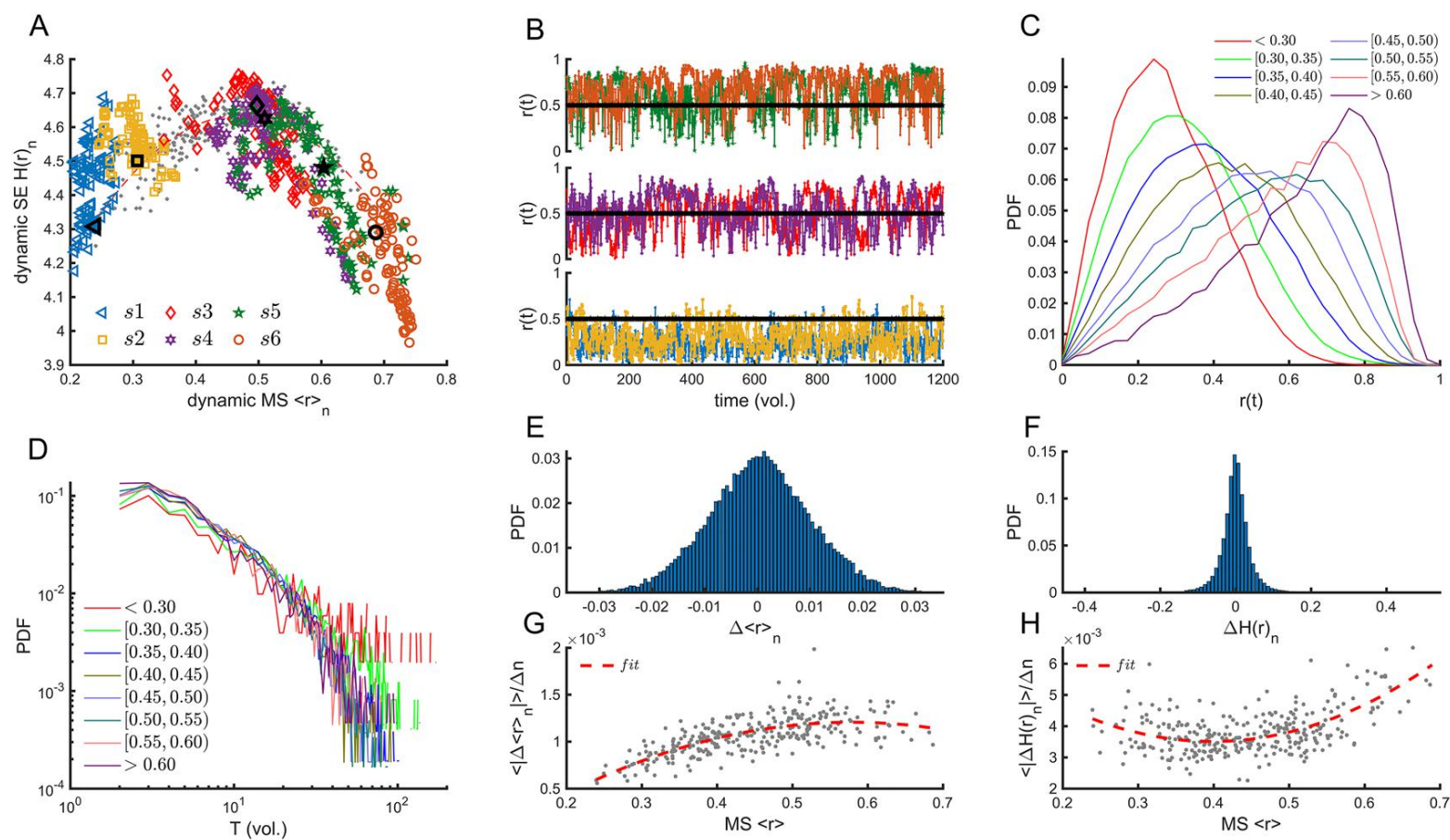

665

Figure 5. The dynamic phase transition in individual subjects' brains. A. The dependence of dynamic SE $H(r)_{n}$ on dynamic MS $\langle r\rangle_{n}$ from six subjects selected randomly from the LMS, MMS, and HMS groups. The enlarged dark markers indicate the mean position for corresponding subjects (markers with the same shape). B. The time-dependent changes in the

670 Kuramoto order parameter $r(t)$ for six subjects as demonstrated in figure a (with the same color). C. The normalized frequency count of $r(t)$ for different levels of $\langle r\rangle$, indicated by 
673 critical point crossing events) distribution for different levels of $\langle r\rangle$. (E, F). The distribution 674 of vertical and horizontal moving distances of phase points in one step of the sliding window.

675 (G, H). The vertical and horizontal velocities of state points of each subject as a function of 676 their MS $\langle r\rangle$. The vertical and horizontal velocities were calculated by $\frac{\left\langle\left|\Delta\langle r\rangle_{n}\right|\right\rangle}{\Delta n}$ and $\frac{\left\langle\left|\Delta\langle r\rangle_{n}\right|\right\rangle}{\Delta n}$, 677 where the symbol $|\cdot|$ indicates the absolute value, and $\langle\cdot\rangle$ was the average across all the 678 windows. $\Delta n$ is the step used to slide the windows. Here, $\Delta \mathrm{n}=10$ time points (volumes). Red 679 dashed lines in G-H: quadratic fitting $\left(F=139.316, p<0.001\right.$, adjusted $R^{2}=0.485$ in $680(\mathrm{G}) ; F=81.181, p<0.001$, adjusted $R^{2}=0.353$ in figure h). Both quadratic fittings were better than linear fitting (adjusted $R^{2}=0.407$ in $\mathbf{G}$ and adjusted $R^{2}=0.173$ in $\mathbf{H}$ ). an opportunity to investigate the dynamic phase transition in individual brains. To this end, for the LMS, MMS, and HMS groups defined above, we randomly selected two subjects from each group. We calculated the dynamical MS $\langle\mathrm{r}\rangle_{\mathrm{n}}$ and $\mathrm{SE} \mathrm{H}(\mathrm{r})_{\mathrm{n}}$ for these six subjects with the sliding window approach (Fig. 5A, s1-s6) from their Kuramoto order parameters r(t) (Fig. 5B). We observed a time-dependent change in individuals' brain states in the state space following the inverted-U trajectory, as shown in the top panel of Fig. 2A. In the time period limited by scan duration, we observed that subjects who were farther away from the critical point tended to stay in the regime decided by MS, and events of crossing the critical point (black lines at $r(t)=0.5$ ) to the other regime seldom occurred $(s 1, s 2, s 5$, and $s 6$ in Fig. 5A, or Fig. 5B, top and 
To validate the above observation at the population level, we divided the 295 subjects at hand into eight groups with different levels of synchrony and calculated the corresponding probability distribution of the Kuramoto order parameter $r(t)$. It is seen clearly from Fig. 5C that as the synchrony level decreases, the distribution of the Kuramoto order parameter becomes narrow and less tilted. Meanwhile, we found that the dwell time, which referred to the time interval between two successive critical point crossing events, exhibited heavier tails in its distribution for low synchrony groups (Fig. 5D). These results implied the higher inertness in the subcritical regime than others, and brains were more likely to stay in this regime with longer dwell times.

Next, we calculated the distribution of vertical and horizontal moving distances in state space in a fixed time interval $\Delta n$ (the time points or volumes of one step of the sliding window) for all subjects. We found that the distributions of vertical and horizontal moving distances were both symmetrical with a mean of zero (Fig. 5E-F), suggesting that the inverted-U trajectory in the state space was stable and unlikely to change its shape as time progressed. Furthermore, the position $(\langle r\rangle)$ dependent velocity distribution is maximal for horizontal velocity

$713\left(\frac{\left\langle\left|\Delta\langle r\rangle_{n}\right|\right\rangle}{\Delta n}\right)$ and minimal for vertical velocity $\left(\frac{\left\langle\left|\Delta\langle r\rangle_{n}\right|\right\rangle}{\Delta n}\right)$ near the critical point (Fig. 5G-H). The maximal horizontal velocity around the critical point implied that at 
716 fluctuations or external modulations. Meanwhile, the lower vertical and

717 horizontal velocities in the subcritical regime compared to the supercritical

718 regime also reflected the high inertness in the subcritical regime.

A

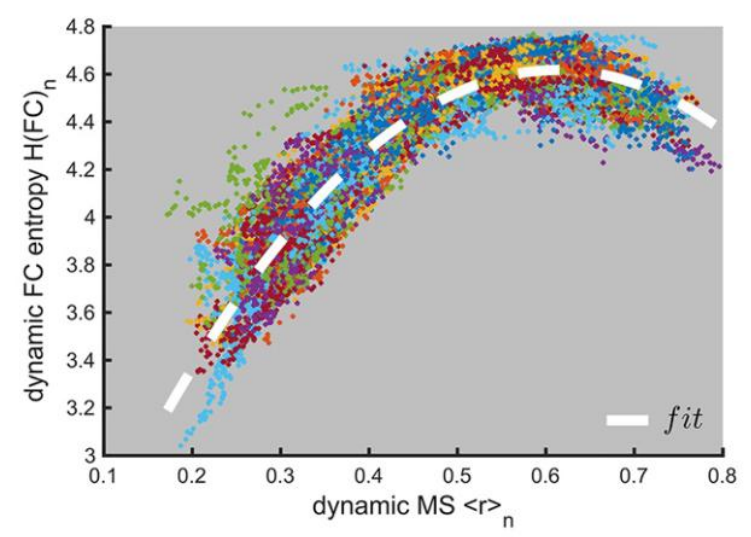

C

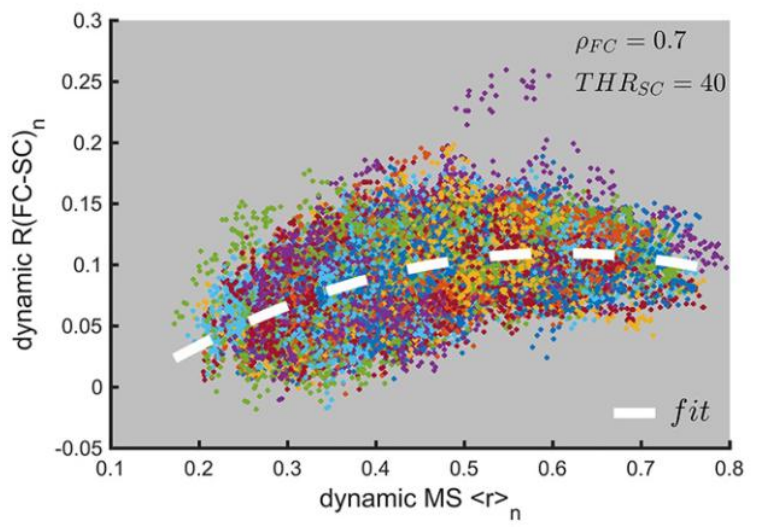

B

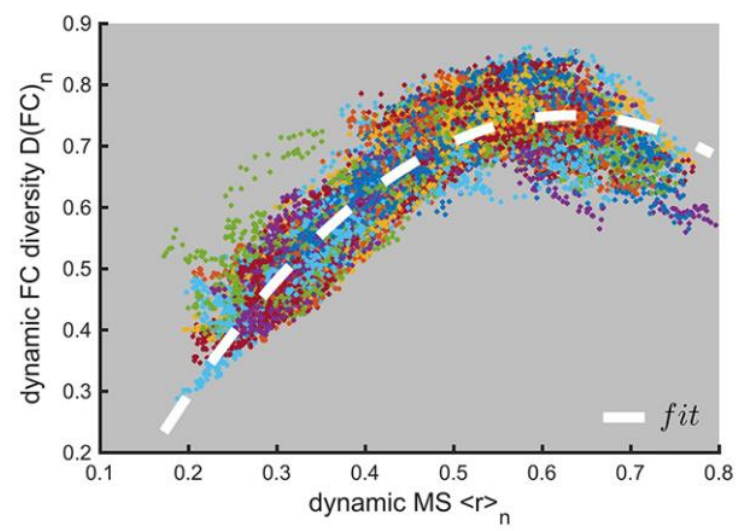

$\mathrm{D}$

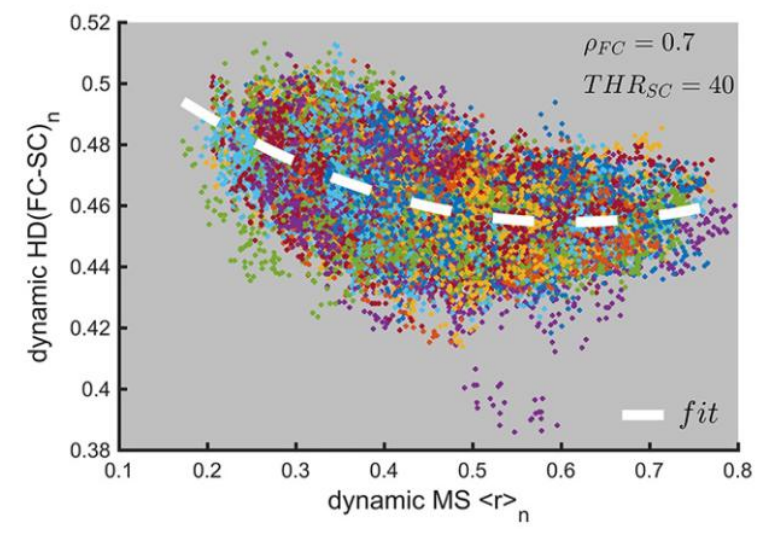

function of instantaneous MS; thick dashed white line: quadratic fitting ( $F=106350.82$, $p<0.001$, adjusted $R^{2}=0.877$ ).

B. The dependence of dynamic FC diversity as a function of instantaneous MS. Thick dashed white line: quadratic fitting $(F=80261.492$, $p<0.001$, adjusted $\left.\mathrm{R}^{2}=0.843\right) . \mathrm{C}$ C. The dependence of dynamic FC-SC correlation as a 
function of instantaneous MS; thick dashed white line: quadratic fitting ( $F=5519.072$,

$727 p<0.001$, adjusted $R^{2}=0.270$ ), which is better than linear fitting (adjusted $R^{2}=0.225$ ).

728 D. The dependence of dynamic FC-SC Hamming distance as a function of instantaneous MS;

729 thick dashed white line: quadratic fitting $(F=5519.072, p<0.001$, adjusted

$730 R^{2}=0.270$ ), which is better than linear fitting (adjusted $R^{2}=0.225$ ). In $\mathbf{A}-\mathbf{D}$, each dot

731 represents a calculation from one window. The dots with the same color represent the

732 calculation for one subject. However, due to the limited number of colors used, different

733 subjects may share the same color. In C-D, a link density of 0.7 was used to obtain the binary

734 FC network, and a threshold of 40 was used to obtain the group-aggregated structural

735 network.

736 It was of interest to determine whether the maximization of FC complexity, as

737 well as function-structure coupling, around the critical point could be realized dynamically when the individual brains endured phase transition. To this end, we obtained the time-dependent FC matrices with the sliding window method and calculated FC entropy (Fig. 6A), FC diversity (Fig. 6B), and two measures

741 for similarity between FC and SC (Fig. 6C-D) as a function of instantaneous MS in each time window. The time-dependent complexity and similarity measures followed almost the exact trajectories as those in the static measurements shown in Fig. 3A-B, as well as Fig. 4A-B. This result implied that FC complexity and similarity between FC and SC were indeed modulated 


\section{High fluid intelligence and working memory capacity were associated with}

\section{critical dynamics}
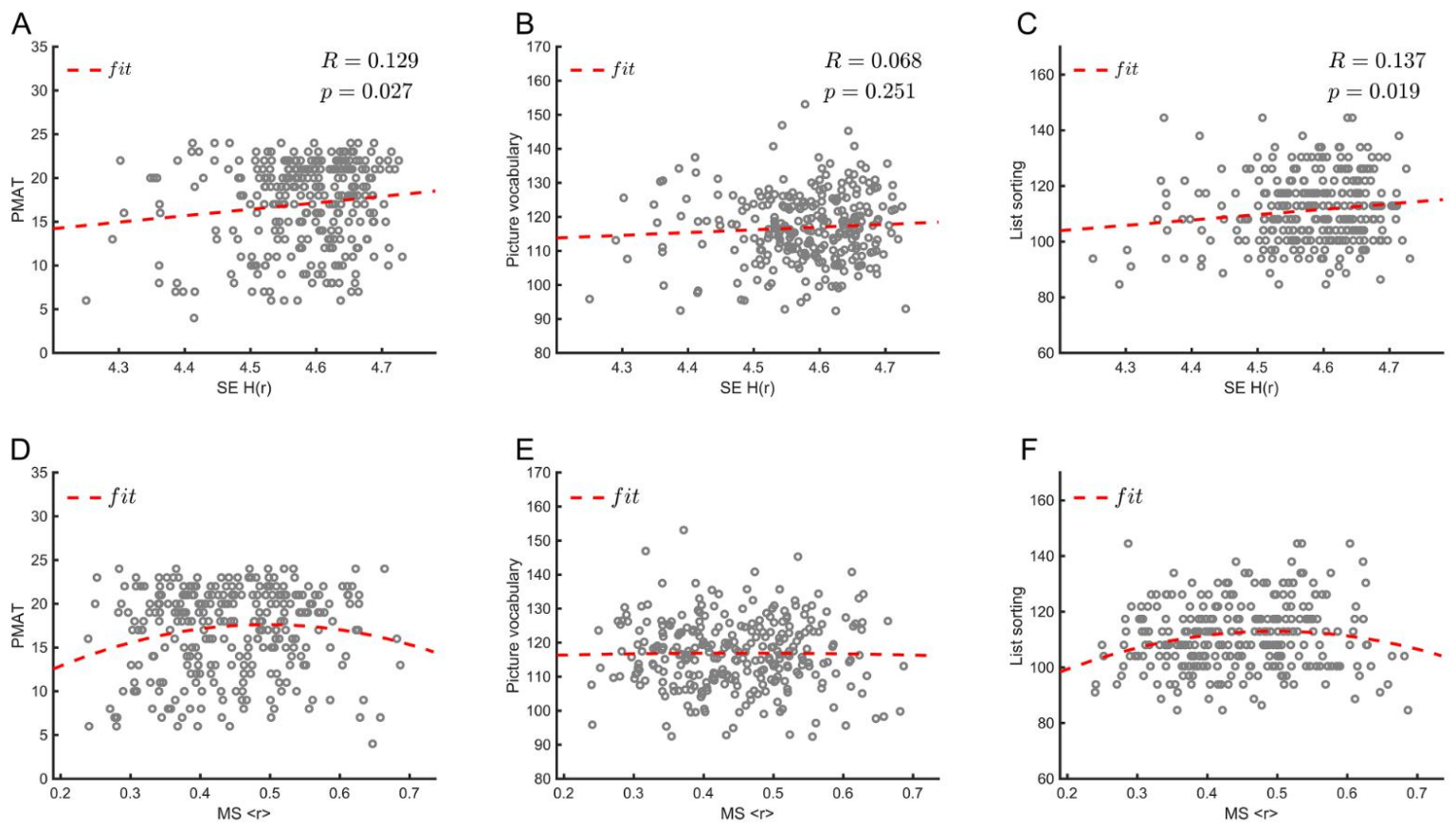

Figure 7. Correlations between cognitive performance scores and SE, as well as MS.

A-C. Correlation between SE and PMAT scores, picture vocabulary test scores, as well as the

list sorting working memory test scores. Red dashed lines in A-C: linear fitting.

D.

Scatterplot of the PMAT scores against the MS. The red dashed line represents the significant quadratic fit of the data $\left(F=3.145, p=0.045\right.$, adjusted $\left.R^{2}=0.015\right)$, which is better than the linear fitting (adjusted $R^{2}=0.004$ ). E. Scatterplot of the picture vocabulary test scores against the MS. Both the linear and quadratic regressions are not significant (linear: $p=$ 
against the MS. The red dashed line represents the significant quadratic fit of the data $(F=$ 4.376, $p=0.013$, adjusted $R^{2}=0.023$ ), which is better than linear fitting (adjusted $R^{2}=$ $0.008)$.

The results above support the hypothesis that large-scale brain networks lie in the vicinity of a critical point which is associated with moderate MS and maximal SE. Another key prediction from the critical brain hypothesis is that brains that are closer to criticality should be better in cognitive performance. Here, to address this prediction, we assessed linear relationships between SE and intelligence scores of the available subjects. We found that SE values were significantly correlated with fluid intelligence scores (PMAT; Fig. 7A) but not with crystallized intelligence scores (picture vocabulary; Fig. 7B). Meanwhile, we found that working memory scores, which were assessed using the Listing Sorting Working Memory test from the NIH Toolbox, were significantly correlated with SE (List sorting; Fig. 7C). We also noted here that these scores were significantly correlated with many other measures that were found to be maximized at the criticality, namely, FC entropy, FC diversity, and FC flexibility (Fig. S11 in Supplementary Materials). Meanwhile, there were significant quadratic relationships between MS and fluid intelligence, as well as working memory scores, but not for crystallized intelligence scores (Fig. 7D-F).

We also found these results still held when potential confounds such as age and education achievements were regressed out (Fig. S12 and S13). Therefore, these 
bioRxiv preprint doi: https://doi org/10.1101/2020 08.24 260588; this version posted August 15, 2021. The copyright holder for this preprint (which was not certified by peer review) is the author/funder, who has granted bioRxiv a license to display the preprint in perpetuity. It is made available under aCC-BY-NC-ND 4.0 International license.

781 fluid intelligence and working memory scores.
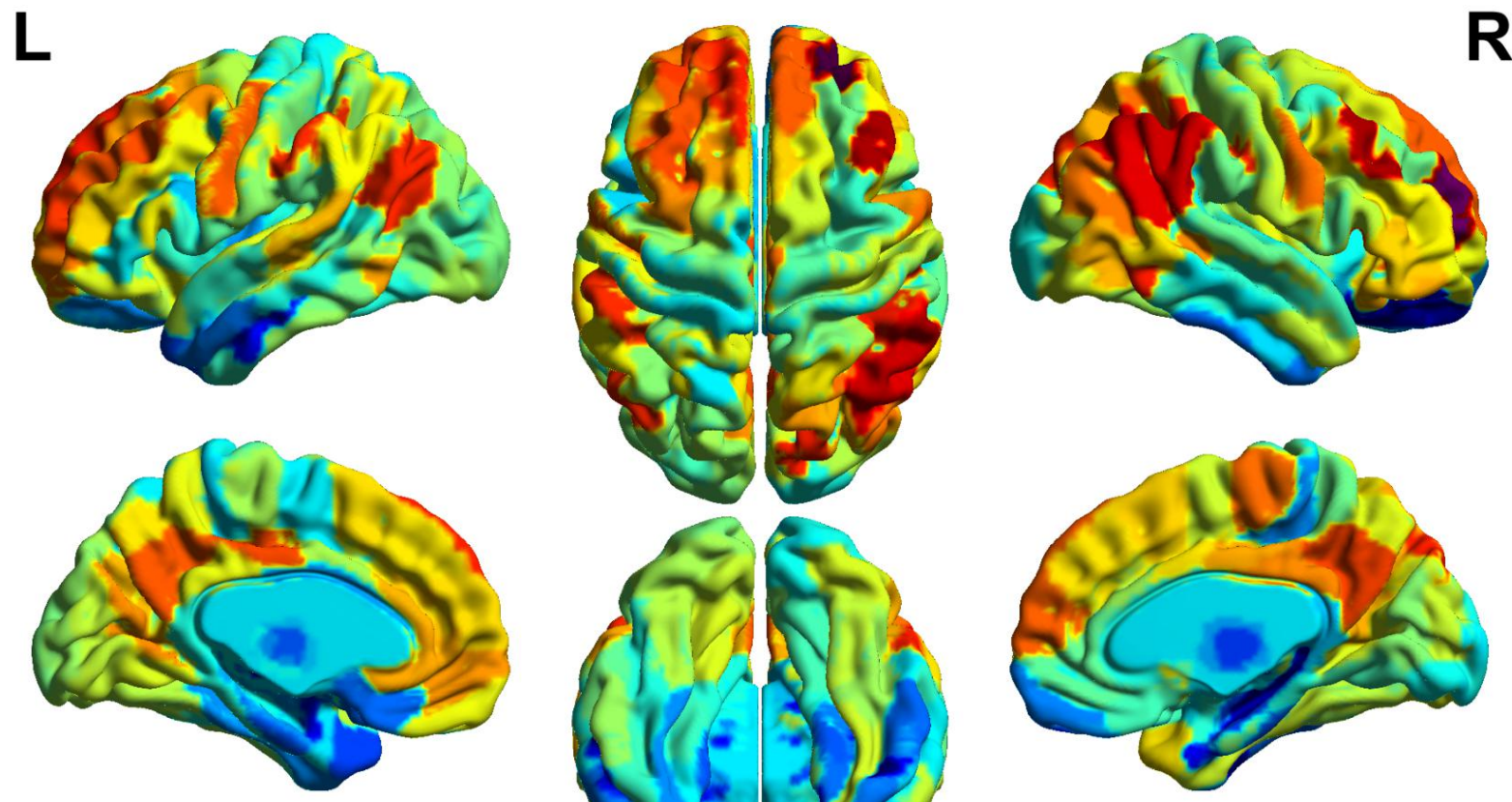

782
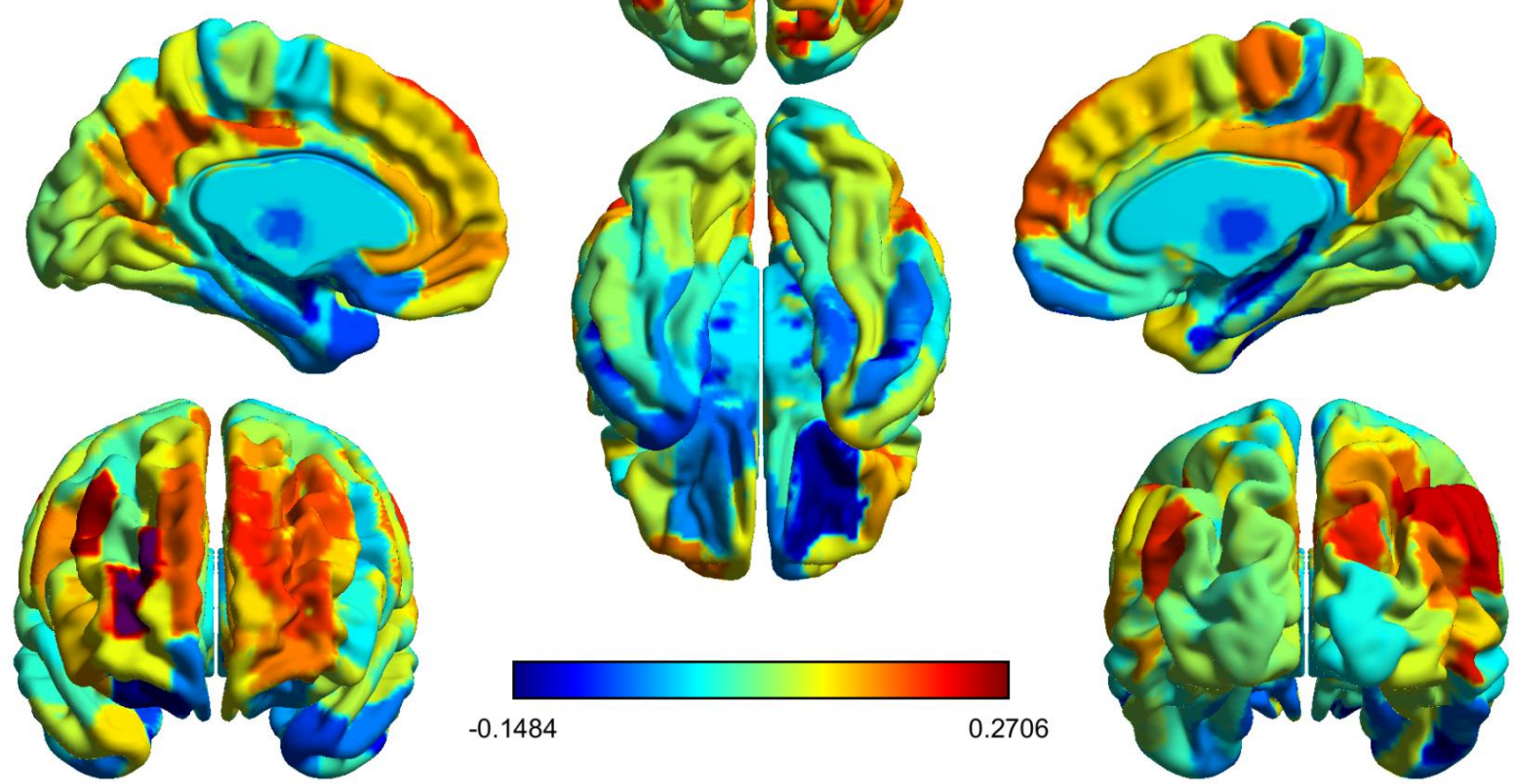

Figure 8. The brain map for correlations between regional SE and fluid intelligence. The

color bar indicated the Pearson correlation value between regional SE and PMAT. The

cortical and subcortical regions were defined by the Human Brainnetome Atlas

(http://atlas.brainnetome.org/bnatlas.html). Data was visualized using BrainNet Viewer (Xia,

787 Wang, \& He, 2013).

Since a wide variety of experiments have demonstrated that fluid intelligence is associated with a distributed network of regions in the Parieto-Frontal 
790 Integration Theory (P-FIT), including frontal areas (Brodmann areas (BAs) 6, 9, 791 10, 45-47), parietal areas (BA 7, 39, 40), visual cortex (BAs 18, 19), fusiform gyrus (BA 37), Wernicke’s area (BA 22) and dorsal anterior cingulate cortex (BA 32) (Jung \& Haier, 2007; Nikolaidis et al., 2017), we decided to find more relationships between these regions with critical dynamics indicated by maximized SE.

796 To obtain the relevant regions in a fine-grained division of the brain, here we used the Human Brainnetome Atlas, which contains 210 cortical and 36 subcortical subregions (Fan et al., 2016). We extracted from each brain region 799 the voxel-level BOLD signals and calculated the regional SE for these 246 800 regions. We found that regions whose SE exhibited significant $(p<0.05$, FDR corrected) positive correlations with PMAT scores were located in the frontal areas (i.e., bilateral SFG, MFG, PrG, right IFG and PCL), parietal areas (i.e., bilateral AG, SMG, Pcun, right SPL), right inferior temporal gyrus (ITG), superior occipital gyrus (sOcG) and left Cingulate gyrus (CG) (Fig 8 and Table 1). 
bioRxiv preprint doi: https://doi org/10.1101/2020.08.24.260588; this version posted August 15,2021 . The copyright holder for this preprint (which was not certified by peer review) is the author/funder, who has granted bioRxiv a license to display the preprint in perpetuity. It is made available under aCC-BY-NC-ND 4.0 International license.

Table 1. The brain regions exhibited significant correlation between $\mathrm{SE}$ and fluid intelligence.

\begin{tabular}{|c|c|c|c|c|c|c|}
\hline Lobe & Gyrus & Left/Right & $\begin{array}{l}\text { Brodmann } \\
\text { area }\end{array}$ & $\begin{array}{c}\text { MNI } \\
\text { coordinate }\end{array}$ & $R$ value & FDR \\
\hline Frontal & Superior Frontal & SFG_L_7_3 & $8,9,10$ & {$[-11,49,40]$} & 0.2058 & 0.0120 \\
\hline \multirow[t]{12}{*}{ Lobe } & Gyrus (SFG) & SFG_R_7_3 & $8,9,10$ & {$[13,48,40]$} & 0.1795 & 0.0264 \\
\hline & & SFG_R_7_7 & 10 & {$[8,58,13]$} & 0.1784 & 0.0268 \\
\hline & Middle Frontal & MFG_L_7_1 & 9,46 & {$[-27,43,31]$} & 0.1827 & 0.0264 \\
\hline & Gyrus (MFG) & MFG_L_7_3 & $10,11,46$ & {$[-28,56,12]$} & 0.1947 & 0.0176 \\
\hline & & MFG_R_7_3 & $9,10,46$ & {$[28,55,17]$} & 0.2706 & 0.0007 \\
\hline & & MFG_L_7_5 & $8,9,44,46$ & {$[-33,23,45]$} & 0.1871 & 0.0229 \\
\hline & & MFG_R_7_5 & $9,44,45,46$ & {$[42,27,39]$} & 0.2522 & 0.0017 \\
\hline & & MFG_L_7_7 & $10,11,47$ & {$[-26,60,-6]$} & 0.1662 & 0.0429 \\
\hline & $\begin{array}{l}\text { Inferior Frontal } \\
\text { Gyrus (IFG) }\end{array}$ & IFG_R_6_5 & 45,47 & {$[42,22,3]$} & 0.1820 & 0.0264 \\
\hline & Precentral Gyrus & PrG_L_6_1 & 4,6 & {$[-49,-8,39]$} & 0.1797 & 0.0264 \\
\hline & $(\operatorname{PrG})$ & PrG_R_6_1 & 4,6 & {$[55,-2,33]$} & 0.1718 & 0.0344 \\
\hline & $\begin{array}{c}\text { Paracentral Lobule } \\
\text { (PCL) }\end{array}$ & PCL_R_2_2 & 4,6 & {$[5,-21,61]$} & 0.1728 & 0.0344 \\
\hline $\begin{array}{l}\text { Tempora } \\
1 \text { Lobe }\end{array}$ & $\begin{array}{c}\text { Inferior Temporal } \\
\text { Gyrus (ITG) }\end{array}$ & ITG_R_7_5 & $20,21,37$ & {$[54,-57,-8]$} & 0.2066 & 0.0120 \\
\hline \multirow[t]{10}{*}{$\begin{array}{l}\text { Parietal } \\
\text { Lobe }\end{array}$} & $\begin{array}{c}\text { Superior Parietal } \\
\text { Lobule (SPL) }\end{array}$ & SPL_R_5_5 & 7,40 & {$[31,-54,53]$} & 0.1717 & 0.0344 \\
\hline & Angular Gyrus & IPL_R_6_1 & 39 & {$[45,-71,20]$} & 0.1687 & 0.0390 \\
\hline & $(\mathrm{AG})$ & IPL_R_6_2 & 7,39 & {$[39,-65,44]$} & 0.2373 & 0.0028 \\
\hline & & IPL_L_6_5 & 39 & {$[-47,-65,26]$} & 0.2011 & 0.0140 \\
\hline & & IPL_R_6_5 & 39 & {$[53,-54,25]$} & 0.2284 & 0.0043 \\
\hline & Supramarginal & IPL_L_6_3 & $2,3,40$ & {$[-51,-33,42]$} & 0.1997 & 0.0140 \\
\hline & Gyrus (SG) & IPL_R_6_3 & $2,3,40$ & {$[47,-35,45]$} & 0.2061 & 0.0120 \\
\hline & & IPL_R_6_4 & 2,40 & {$[57,-44,38]$} & 0.2369 & 0.0028 \\
\hline & Precuneus (Pcun) & Pcun_L_4_4 & 7 & {$[-6,-55,34]$} & 0.1810 & 0.0264 \\
\hline & & Pcun_R_4_4 & 7 & {$[6,-54,35]$} & 0.1899 & 0.0219 \\
\hline $\begin{array}{l}\text { Limbic } \\
\text { Lobe }\end{array}$ & $\begin{array}{l}\text { Cingulate Gyrus } \\
\text { (CG) }\end{array}$ & CG_L_7_6 & 23 & {$[-7,-23,41]$} & 0.1868 & 0.0229 \\
\hline $\begin{array}{l}\text { Occipital } \\
\text { Lobe }\end{array}$ & $\begin{array}{l}\text { Superior Occipital } \\
\text { Gyrus (sOcG) }\end{array}$ & sOcG_R_2_1 & 18,19 & {$[16,-85,34]$} & 0.2051 & 0.0120 \\
\hline
\end{tabular}

812 Abbreviation: IPL, Inferior Parietal Lobule.

813 Discussion

814 In this study, using the large scale WU-Minn HCP dataset and a large number 
815 of criticality-inspired metrics, we provided evidence that though healthy young

816 brains at rest are on average close to the critical point, as critical brain

817 hypothesis has suggested, there is a considerable individual variation around

818 this point. This gave us a chance to validate previous theoretical predictions of

819 criticality on large scale brain networks, and indeed we observed that the

820 complexity of brain FC, as well as the structure-function coupling, was

maximized around the critical point. We proceeded to observe a dynamic phase

transition in individual subjects, and found that their brains tended to stay subcritical, as indicated by a longer dwell time in this parameter region. Finally, we found that high fluid intelligence and working memory capacity were associated with critical dynamics rather than noncritical dynamics, not only globally but also regionally, suggesting the functional advantages of critical dynamics in resting-state brains.

Balance between functional segregation and functional integration is a central organizing principle of the cerebral cortex. It has been argued that FC

830 complexity characterizes the interplay of functional segregation and functional integration (Sporns, 2013). A comparison between simulated and empirically 832 obtained resting-state FC indicates that the human brain at rest lies in a dynamic state that reflects the largest complexity its anatomical connectome can host (G.

834 Tononi, Sporns, \& Edelman, 1994). Recently, many studies have tried to link 835 complexity with cognitive performance, human intelligence, and even 
838 Chylinski, Mitchell, \& Bhandari, 2017; Saxe, Calderone, \& Morales, 2018;

839 Giulio Tononi, Edelman, \& Sporns, 1998). Meanwhile, there is a growing awareness that complexity is strongly related to criticality. A recent study 841 showed that criticality maximized complexity in dissociated hippocampal 842 cultures produced from rats (Timme et al., 2016). Here, in this study, we measured FC complexity from different perspectives, either on its strength 844 diversity or on its dynamic flexibility (Fig. 3A-C, Fig. 6A-B). With the observation of the phase transition trajectory, we demonstrated that these measures of FC complexity were maximized around the critical point. Therefore, the formulation that criticality maximizes complexity was supported in our work empirically with fMRI data at the whole-brain network level.

It has been shown that human brains possess a stable set of functionally coupled networks that echo many known features of anatomical organization (Krienen, Yeo, \& Buckner, 2014). Several computational modeling studies have demonstrated that critical dynamics could best explore the repertoire provided by the structural connectome (Deco \& Jirsa, 2012; Enzo Tagliazucchi et al., 854 2016). Recent studies also suggested the capacity of repertoire provided by the 855 structural connectome could be extended by the hierarchical modular structural organization (R. Wang et al., 2019). Therefore, structure-function coupling was 
857

858

859

860

861

believed to be at its maximal when the system is at criticality (R. Wang et al., 2019), and it could be disrupted by losing criticality (Cocchi et al., 2014), or disruption of hierarchical organization of structural networks. Previous studies in anesthetized human brains have found structure-function decoupling accompanied by unidirectional departure from a critical point (Enzo Tagliazucchi et al., 2016). It is possible that functional connectivity flexibility could be used as a measure of the extent that functional connectivity explores the repertoire provided by structural connectome, and the highest functional connectivity flexibility occurs when the system is at criticality (Song et al., 2019) (Fig. 3C). Our work demonstrated the maximal exploration of structural connections at the critical point occurs in resting state brains (Fig. 4). However, since we used a group aggregated structural connection networks, we did not investigate how organization of structural connections could impact on the capacity of network repertoire. This issue will be investigated in the future.

Interestingly, although the brain hovers around the critical point, the brain prefers to stay in the subcritical region, as the subject distribution was skewed toward a disordered state, and the dwell time in the subcritical state was longer (Fig. 5). Previous analysis of in vivo data has argued that the mammalian brain self-organizes to a slightly subcritical regime (Priesemann et al., 2014). It was suggested that operating in a slightly subcritical regime may prevent the brain from tipping over to supercriticality, which has been linked to epilepsy. 
878

879 the computational capabilities may still be close to optimal. However, our 880 results showed that the resting state brains could actually stay in the 881 supercritical regimes. So, the preference of brains for subcritical regime may

Meanwhile, with a slightly subcritical regime deviates only little from criticality, not because of prevention of too ordered states. In another study, by relating the EEG-domain cascades to spatial BOLD patterns in simultaneously recorded fMRI data, the researchers found that while resting-state cascades were associated with an approximate power-law form, the task state was associated with subcritical dynamics (Fagerholm et al., 2015). They argued that while a high dynamic range and a large repertoire of brain states may be advantageous for the resting state with near-critical dynamics, a lower dynamic range may reduce elements of interference affecting task performance in a focused cognitive task with subcritical dynamics (Fagerholm et al., 2015). Therefore, there remains a possibility that the resting state is not "pure resting state", but mixed with some occasional "task state" for some subjects. However, further delicately designed experimental studies are required to test this conjecture. It remains to uncover the relationship between cognitive states and neural dynamics that lies on a spectrum. The method proposed in this study may be useful in future studies of this topic.

Recently, Ezaki et al. used the Ising model to map BOLD signals on a two-dimensional phase space and found that human fMRI data were in the 
paramagnetic phase and were close to the boundary with the spin-glass phase

900 but not to the boundary with the ferromagnetic phase (Ezaki, Fonseca dos Reis, 901 Watanabe, Sakaki, \& Masuda, 2020). Since the spin-glass phase usually yields 902 chaotic dynamics whereas the ferromagnetic phase is nonchaotic, their results suggested that the brain is around the "edge of chaos criticality" instead of "avalanche criticality". However, our findings support that avalanche criticality occur in large-scale brain networks. Therefore, it is interesting to investigate whether both kinds of criticality could co-occur in large-scale brain networks (Kanders et al., 2017). Ezaki et al. also found that criticality of brain dynamics was associated with human fluid intelligence, though they used performance IQ to reflect fluid intelligence, which refers to active or effortful problem solving and maintenance of information. In our work, we assessed the correlation between fluid intelligence and the critical dynamics indicated by synchronization entropy for brain regions, and found regions showed significant positive correlations were located in parietal-frontal network (Fig. 8 and Table 1). These regions were most frequently reported in studies of intelligence and its biological basis, including structural neuroimaging studies using voxel-based morphometry, magnetic resonance spectroscopy, and DTI, as well as functional 917 imaging studies using positron emission tomography (PET) or fMRI (Jung \& 918 Haier, 2007). Also, in the Parieto-Frontal Integration Theory of intelligence, 919 these regions are considered as the most crucial nodes of the brain network 
underlying human intelligence (Jung \& Haier, 2007; Nikolaidis et al., 2017).

921 Our study suggested that not only fluid intelligence, but also working memory capacity was associated with critical dynamics. This is possibly because working memory may share the same capacity constraint through similar neural networks with fluid intelligence (Halford, Cowan, \& Andrews, 2007; Jaeggi, Buschkuehl, Jonides, \& Perrig, 2008; Kane \& Engle, 2002). In our study, the critical dynamics in the frontal and parietal network also exhibited significant correlation with working memory capacity (Fig. S14 and Table S1). Furthermore, it has been well established that working memory is strongly modulated by dopamine, and too strong or too weak dopamine D1 activation is detrimental for working memory, with the optimal performance achieved at an 931 intermediate level (Cools \& D'Esposito, 2011; Vijayraghavan, Wang, Birnbaum, 932 Williams, \& Arnsten, 2007; Zahrt, Taylor, Mathew, \& Arnsten, 1997). This inverted-U dose-response has been observed in mice (Lidow, Koh, \& Arnsten, 934 2003), rats (Zahrt et al., 1997), monkeys (Cai \& Arnsten, 1997) and humans 935 (Gibbs \& D'Esposito, 2005). Recent studies on neural network models have 936 shown that the optimal performance of working memory co-occurs with critical 937 dynamics at the network level and the excitation-inhibition balance at the level 938 of individual neurons and is modulated by dopamine at the synaptic level 939 through a series of $U$ or inverted-U profiles (Hu, Huang, Jiang, \& Yu, 2019). 940 Here in this study, we demonstrated that the optimal performance of working 
memory and criticality co-occurs at the system level.

942 However, our study had several limitations. Firstly, the surrogate data test used 943 in this study ruled out the possibility that the results we obtained could be 944 explained by autocorrelations in the data. However, the long-range spatial 945 correlation of criticality cannot allow one to test the results by ruling out the 946 effects of correlation across the time series. Secondly, though we used the 947 denoising fMRI data from HCP with standard data pre-processing procedure, it 948 is still interesting to investigate how the pre-processing procedure affects the 949 results. Thirdly, in the avalanche analysis, the activation events defined in this 950 study were slightly different from definition used by others, such as threshold-crossing events (Enzo Tagliazucchi et al., 2012) or above-threshold 952 events (Bocaccio et al., 2019; R. Wang et al., 2019). We compared these 953 different methods and found all these methods could generate scale free 954 avalanche activities, but unlike our method, the other two methods failed to generate critical branching process (See Section II in Supplementary Materials). 956 Therefore, it is interesting to investigate the correlations between neural 957 activities and events detected by different detection methods from BOLD signals. Finally, in this study we only focused on the cognitive abilities that are associated with critical dynamics, and found significant but not strong 960 correlations between fluid intelligence, working memory and critical dynamics. 961 Recent works demonstrated that the functional network segregation, integration 
and their balance could predict different cognitive abilities (R. Wang et al., 963 2021). Therefore, future investigation on the relationship between this 964 functional balance and criticality across individuals may reveal various 965 associations between diverse cognitive abilities with not only critical dynamics, 966 but also non-critical dynamics. Despite all these shortages, we hope this study 967 may inspire future research work to validate our findings, e.g., through 968 observing not only the association between the departure of criticality and the 969 decline of cognitive performance, either in aging or brain disease, but also the 970 restore of criticality and the improvement of cognitive performance with 971 pharmacological or noninvasive brain stimulation (Barch, 2004; Reinhart \& 972 Nguyen, 2019).

\section{${ }_{973}$ Conclusions}

974 In conclusion, we mapped individuals' brain dynamics from resting-state fMRI 975 scans on the phase transition trajectory and identify subjects who are close to 976 the critical point. With this approach, we validated two predictions of critical 977 brain hypothesis on large-scale brain networks, i.e., maximized FC complexity 978 and maximized structure-function coupling around the critical point. We also 979 observed the tendency of brain to stay in subcritical regime. Finally, we found 980 that the critical dynamics in large-scale brain networks were associated with 981 high scores in fluid intelligence and working memory, implying the vital role of 982 large-scale critical dynamics in cognitive performance. We also identified key 
brain regions whose critical dynamics was highly correlated with human intelligence. Our findings support the critical brain hypothesis that neural computation is optimized by critical brain dynamics, as characterized by scale-free avalanche activity, and could provide a solution for improving the effects of future interventions targeting aspects of cognitive decline (Reinhart \&

Nguyen, 2019), possibly by controlling the criticality through non-invasive stimulation (Chialvo, Cannas, Grigera, Martin, \& Plenz, 2020).

\section{Conflicts of interest}

The authors declare no competing interests.

\section{$992 \quad$ Funding}

This study was funded by the National Natural Science Foundation of China

Universities (Grant No. lzujbky-2021-62). J. F. is supported by the 111 Project

996

(Grant No. B18015), the National Key R\&D Program of China

(No.2018YFC1312904; No.2019YFA0709502), the Shanghai Municipal

Science and Technology Major Project (Grant No. 2018SHZDZX01), ZJLab,

\section{References}

Ahmadlou, M., Adeli, A., Bajo, R., \& Adeli, H. (2014). Complexity of functional connectivity networks in mild cognitive impairment subjects during a working memory task.

Clinical Neurophysiology,

125(4),

694-702. doi:https://doi.org/10.1016/j.clinph.2013.08.033 
1005

1006

1007

1008

1009

1010

1011

1012

1013

1014

1015

1016

1017

1018

1019

1020

1021

1022

1023

1024

1025

1026

1027

1028

1029

1030

1031

1032

1033

1034

1035

1036

1037

1038

1039

Anokhin, A. P., Birbaumer, N., Lutzenberger, W., Nikolaev, A., \& Vogel, F. (1996). Age increases brain complexity. Electroencephalography and Clinical Neurophysiology, 99(1), 63-68. doi:https://doi.org/10.1016/0921-884X(96)95573-3

Barch, D. M. (2004). Pharmacological manipulation of human working memory. Psychopharmacology, 174(1), 126-135. doi:10.1007/s00213-003-1732-3

Barch, D. M., Burgess, G. C., Harms, M. P., Petersen, S. E., Schlaggar, B. L., Corbetta, M., . . . Consortium, W. U.-M. H. (2013). Function in the human connectome: task-fMRI and individual differences in behavior. NeuroImage, 80, 169-189. doi:10.1016/j.neuroimage.2013.05.033

Beggs, J., \& Timme, N. (2012). Being Critical of Criticality in the Brain. Frontiers in Physiology, 3, 163.

Beggs, J. M., \& Plenz, D. (2003). Neuronal avalanches in neocortical circuits. The Journal of neuroscience : the official journal of the Society for Neuroscience, 23(35), 11167-11177. doi:10.1523/JNEUROSCI.23-35-11167.2003

Bilker, W. B., Hansen, J. A., Brensinger, C. M., Richard, J., Gur, R. E., \& Gur, R. C. (2012). Development of Abbreviated Nine-Item Forms of the Raven's Standard Progressive Matrices Test. Assessment, 19(3), 354-369. doi:10.1177/1073191112446655

Bocaccio, H., Pallavicini, C., Castro, M. N., Sánchez, S. M., De Pino, G., Laufs, H., . . . Tagliazucchi, E. (2019). The avalanche-like behaviour of large-scale haemodynamic activity from wakefulness to deep sleep. Journal of The Royal Society Interface, 16(158), 20190262. doi:10.1098/rsif.2019.0262

Cai, J. X., \& Arnsten, A. F. T. (1997). Dose-Dependent Effects of the Dopamine D1 Receptor Agonists A77636 or SKF81297 On Spatial Working Memory in Aged Monkeys. Journal of Pharmacology and Experimental Therapeutics, 283(1), 183.

Chialvo, D. R., Cannas, S. A., Grigera, T. S., Martin, D. A., \& Plenz, D. (2020). Controlling a complex system near its critical point via temporal correlations. Scientific Reports, 10(1), 12145. doi:10.1038/s41598-020-69154-0

Clauset, A., Shalizi, C. R., \& Newman, M. E. J. (2009). Power-Law Distributions in Empirical Data. SIAM Review, 51(4), 661-703.

Cocchi, L., Gollo, L. L., Zalesky, A., \& Breakspear, M. (2017). Criticality in the brain: A synthesis of neurobiology, models and cognition. Progress in Neurobiology, 158, 132-152. doi:https://doi.org/10.1016/j.pneurobio.2017.07.002

Cocchi, L., Harding, I. H., Lord, A., Pantelis, C., Yucel, M., \& Zalesky, A. (2014). Disruption of structure-function coupling in the schizophrenia connectome. NeuroImage: Clinical, 4, 779-787. doi:https://doi.org/10.1016/j.nicl.2014.05.004 
1040

1041

1042

1043

1044

1045

1046

1047

1048

1049

1050

1051

1052

1053

1054

1055

1056

1057

1058

1059

1060

1061

1062

1063

1064

1065

1066

1067

1068

1069

1070

1071

1072

1073

Cools, R., \& D'Esposito, M. (2011). Inverted-U Shaped Dopamine Actions on Human Working Memory and Cognitive Control. Biological Psychiatry, 69(12), e113-e125. doi:10.1016/j.biopsych.2011.03.028

Deco, G., \& Jirsa, V. K. (2012). Ongoing Cortical Activity at Rest: Criticality, Multistability, and Ghost Attractors. The Journal of Neuroscience, 32(10), 3366. doi:10.1523/JNEUROSCI.2523-11.2012

Deco, G., Jirsa, V. K., \& McIntosh, A. R. (2011). Emerging concepts for the dynamical organization of resting-state activity in the brain. Nature Reviews Neuroscience, 12(1), 43-56. doi:10.1038/nrn2961

Deluca, A., \& Corral, Á. (2013). Fitting and goodness-of-fit test of non-truncated and truncated power-law distributions. Acta Geophysica, 61(6), 1351-1394. doi:10.2478/s11600-013-0154-9

Destexhe, A., \& Touboul, J. (2020). Comment on "Criticality Between Cortical States". arXiv: Neurons and Cognition.

Duncan, J., Chylinski, D., Mitchell, D. J., \& Bhandari, A. (2017). Complexity and compositionality in fluid intelligence. Proceedings of the National Academy of Sciences, 114(20), 5295. doi:10.1073/pnas.1621147114

Ezaki, T., Fonseca dos Reis, E., Watanabe, T., Sakaki, M., \& Masuda, N. (2020). Closer to critical resting-state neural dynamics in individuals with higher fluid intelligence. Communications Biology, 3(1), 52. doi:10.1038/s42003-020-0774-y

Fagerholm, E. D., Lorenz, R., Scott, G., Dinov, M., Hellyer, P. J., Mirzaei, N., . . L Leech, R. (2015). Cascades and cognitive state: focused attention incurs subcritical dynamics. The Journal of neuroscience : the official journal of the Society for Neuroscience, 35(11), 4626-4634. doi:10.1523/JNEUROSCI.3694-14.2015

Fan, L., Li, H., Zhuo, J., Zhang, Y., Wang, J., Chen, L., . . . Jiang, T. (2016). The Human Brainnetome Atlas: A New Brain Atlas Based on Connectional Architecture. Cerebral Cortex, 26(8), 3508-3526. doi:10.1093/cercor/bhw157

Fontenele, A. J., de Vasconcelos, N. A. P., Feliciano, T., Aguiar, L. A. A., Soares-Cunha, C., Coimbra, B., . . . Copelli, M. (2019). Criticality between Cortical States. Physical Review Letters, 122(20), 208101. doi:10.1103/PhysRevLett.122.208101

Friedman, N., Ito, S., Brinkman, B. A. W., Shimono, M., DeVille, R. E. L., Dahmen, K. A., . . . Butler, T. C. (2012). Universal Critical Dynamics in High Resolution Neuronal Avalanche Data. Physical Review Letters, 108(20), 208102. doi:10.1103/PhysRevLett.108.208102 
1074

1075

1076

1077

1078

1079

1080

1081

1082

1083

1084

1085

1086

1087

1088

1089

1090

1091

1092

1093

1094

1095

1096

1097

1098

1099

1100

1101

1102

1103

1104

1105

1106

Gal, A., \& Marom, S. (2013). Self-organized criticality in single-neuron excitability. Physical Review E, 88(6), 062717. doi:10.1103/PhysRevE.88.062717

Gibbs, S. E. B., \& D'Esposito, M. (2005). A functional MRI study of the effects of bromocriptine, a dopamine receptor agonist, on component processes of working memory. Psychopharmacology, 180(4), 1-10. doi:10.1007/s00213-005-0077-5

Gireesh, E. D., \& Plenz, D. (2008). Neuronal avalanches organize as nested theta- and beta/gamma-oscillations during development of cortical layer 2/3. Proceedings of the National Academy of Sciences, 105(21), 7576. doi:10.1073/pnas.0800537105

Glasser, M. F., Smith, S. M., Marcus, D. S., Andersson, J. L. R., Auerbach, E. J., Behrens, T. E. J., . . . Van Essen, D. C. (2016). The Human Connectome Project's neuroimaging approach. Nature Neuroscience, 19(9), 1175-1187. doi:10.1038/nn.4361

Glasser, M. F., Sotiropoulos, S. N., Wilson, J. A., Coalson, T. S., Fischl, B., Andersson, J. L., . . J Jenkinson, M. (2013). The minimal preprocessing pipelines for the Human Connectome Project. $\quad$ NeuroImage, $\quad$ 80, doi:https://doi.org/10.1016/j.neuroimage.2013.04.127

Halford, G. S., Cowan, N., \& Andrews, G. (2007). Separating cognitive capacity from knowledge: a new hypothesis. Trends in Cognitive Sciences, 11(6), 236-242. doi:10.1016/j.tics.2007.04.001

Harris, T. E. (1964). The theory of branching process. New York: Dover.

He, B. J. (2011). Scale-free properties of the functional magnetic resonance imaging signal during rest and task. The Journal of neuroscience : the official journal of the Society for Neuroscience, 31(39), 13786-13795. doi:10.1523/JNEUROSCI.2111-11.2011

Hearne, L. J., Mattingley, J. B., \& Cocchi, L. (2016). Functional brain networks related to individual differences in human intelligence at rest. Scientific Reports, 6(1), 32328. doi:10.1038/srep32328

Hesse, J., \& Gross, T. (2014). Self-organized criticality as a fundamental property of neural systems. Frontiers in Systems Neuroscience, 8, 166-166. doi:10.3389/fnsys.2014.00166

Hobbs, J. P., Smith, J. L., \& Beggs, J. M. (2010). Aberrant Neuronal Avalanches in Cortical Tissue Removed From Juvenile Epilepsy Patients. Journal of Clinical Neurophysiology, 27(6).

Hu, G., Huang, X., Jiang, T., \& Yu, S. (2019). Multi-Scale Expressions of One Optimal State Regulated by Dopamine in the Prefrontal Cortex. Frontiers in Physiology, 10, 113-113. doi:10.3389/fphys.2019.00113 
Jaeggi, S. M., Buschkuehl, M., Jonides, J., \& Perrig, W. J. (2008). Improving fluid intelligence with training on working memory. Proceedings of the National Academy of Sciences, 105(19), 6829. doi:10.1073/pnas.0801268105

Jung, R. E., \& Haier, R. J. (2007). The Parieto-Frontal Integration Theory (P-FIT) of intelligence: Converging neuroimaging evidence. Behavioral and Brain Sciences, 30(2), 135-154. doi:10.1017/S0140525X07001185

Kanders, K., Lee, H., Hong, N., Nam, Y., \& Stoop, R. (2020). Fingerprints of a second order critical line in developing neural networks. Communications Physics, 3(1), 13. doi:10.1038/s42005-019-0276-8

Kanders, K., Lorimer, T., \& Stoop, R. (2017). Avalanche and edge-of-chaos criticality do not necessarily co-occur in neural networks. Chaos: An Interdisciplinary Journal of Nonlinear Science, 27(4), 047408. doi:10.1063/1.4978998

Kane, M. J., \& Engle, R. W. (2002). The role of prefrontal cortex in working-memory capacity, executive attention, and general fluid intelligence: An individual-differences perspective. Psychonomic Bulletin \& Review, 9(4), 637-671. doi:10.3758/BF03196323

Karahanoğlu, F. I., \& Van De Ville, D. (2017). Dynamics of large-scale fMRI networks: Deconstruct brain activity to build better models of brain function. Current Opinion in Biomedical Engineering, 3, 28-36. doi:https://doi.org/10.1016/j.cobme.2017.09.008

Krienen, F. M., Yeo, B. T. T., \& Buckner, R. L. (2014). Reconfigurable task-dependent functional coupling modes cluster around a core functional architecture. Philosophical transactions of the Royal Society of London. Series B, Biological sciences, 369(1653), 20130526. doi:10.1098/rstb.2013.0526

Lee, H., Golkowski, D., Jordan, D., Berger, S., Ilg, R., Lee, J., . . . Vlisides, P. E. (2019). Relationship of critical dynamics, functional connectivity, and states of consciousness in large-scale human brain networks. NeuroImage, 188, 228-238. doi:https://doi.org/10.1016/j.neuroimage.2018.12.011

Lei, Y., Song, B., Chen, L., Su, J., Zhang, X., Ni, W., . . . Mao, Y. (2020). Reconfigured functional network dynamics in adult moyamoya disease: a resting-state fMRI study. Brain Imaging and Behavior, 14(3), 715-727. doi:10.1007/s11682-018-0009-8

Lidow, M. S., Koh, P.-O., \& Arnsten, A. F. T. (2003). D1 dopamine receptors in the mouse prefrontal cortex: Immunocytochemical and cognitive neuropharmacological analyses. Synapse, 47(2), 101-108. doi:10.1002/syn.10143

Liu, T. T., Nalci, A., \& Falahpour, M. (2017). The global signal in fMRI: Nuisance or Information? NeuroImage, 150 213-229. doi:https://doi.org/10.1016/j.neuroimage.2017.02.036 
Makris, N., Goldstein, J. M., Kennedy, D., Hodge, S. M., Caviness, V. S., Faraone, S. V., . . . Seidman, L. J. (2006). Decreased volume of left and total anterior insular lobule in schizophrenia. Schizophrenia Research, 83(2), 155-171. doi:https://doi.org/10.1016/j.schres.2005.11.020

Marshall, N., Timme, N. M., Bennett, N., Ripp, M., Lautzenhiser, E., \& Beggs, J. M. (2016). Analysis of Power Laws, Shape Collapses, and Neural Complexity: New Techniques and MATLAB Support via the NCC Toolbox. Frontiers in Physiology, 7, 250.

Meisel, C., Olbrich, E., Shriki, O., \& Achermann, P. (2013). Fading Signatures of Critical Brain Dynamics during Sustained Wakefulness in Humans. The Journal of Neuroscience, 33(44), 17363. doi:10.1523/JNEUROSCI.1516-13.2013

Meisel, C., Storch, A., Hallmeyer-Elgner, S., Bullmore, E., \& Gross, T. (2012). Failure of Adaptive Self-Organized Criticality during Epileptic Seizure Attacks. PLoS Computational Biology, 8(1), e1002312. doi:10.1371/journal.pcbi.1002312

Nakagawa, T. T., Jirsa, V. K., Spiegler, A., McIntosh, A. R., \& Deco, G. (2013). Bottom up modeling of the connectome: Linking structure and function in the resting brain and their $\begin{array}{lllll}\text { changes in } \quad \text { aging. } & \text { NeuroImage, } & \text { 80, } & \text { 318-329. }\end{array}$ doi:https://doi.org/10.1016/j.neuroimage.2013.04.055

Nikolaidis, A., Baniqued, P. L., Kranz, M. B., Scavuzzo, C. J., Barbey, A. K., Kramer, A. F., \& Larsen, R. J. (2017). Multivariate Associations of Fluid Intelligence and NAA. Cerebral Cortex, 27(4), 2607-2616. doi:10.1093/cercor/bhw070

Omidvarnia, A., Zalesky, A., Van De Ville, D., Jackson, G. D., \& Pedersen, M. (2019). Temporal complexity of fMRI is reproducible and correlates with higher order cognition. bioRxiv, 770826. doi:10.1101/770826

Palva, J. M., Zhigalov, A., Hirvonen, J., Korhonen, O., Linkenkaer-Hansen, K., \& Palva, S. (2013). Neuronal long-range temporal correlations and avalanche dynamics are correlated with behavioral scaling laws. Proceedings of the National Academy of Sciences of the United States of America, 110(9), 3585-3590. doi:10.1073/pnas.1216855110

Plenz, D. (2012). Neuronal avalanches and coherence potentials. The European Physical Journal Special Topics, 205(1), 259-301. doi:10.1140/epjst/e2012-01575-5

Poil, S.-S., Hardstone, R., Mansvelder, H. D., \& Linkenkaer-Hansen, K. (2012). Critical-State Dynamics of Avalanches and Oscillations Jointly Emerge from Balanced Excitation/Inhibition in Neuronal Networks. The Journal of Neuroscience, 32(29), 9817. doi:10.1523/JNEUROSCI.5990-11.2012 
Popiel, N. J. K., S.; Abeyasinghe, P.M.; Riganello, F.; Nichols, E.S.; Owen, A.M.; Soddu, A. (2020). The Emergence of Integrated Information, Complexity, and 'Consciousness' at Criticality. Entropy, 22.

Prichard, D., \& Theiler, J. (1994). Generating surrogate data for time series with several simultaneously measured variables. Physical Review Letters, 73(7), 951-954. doi:10.1103/PhysRevLett.73.951

Priesemann, V., Wibral, M., Valderrama, M., Pröpper, R., Le Van Quyen, M., Geisel, T., .. . Munk, M. H. J. (2014). Spike avalanches in vivo suggest a driven, slightly subcritical brain state. Frontiers in Systems Neuroscience, 8, 108-108. doi:10.3389/fnsys.2014.00108

Reinhart, R. M. G., \& Nguyen, J. A. (2019). Working memory revived in older adults by synchronizing rhythmic brain circuits. Nature Neuroscience, 22(5), 820-827. doi:10.1038/s41593-019-0371-x

Sakoğlu, Ü., Pearlson, G. D., Kiehl, K. A., Wang, Y. M., Michael, A. M., \& Calhoun, V. D. (2010). A method for evaluating dynamic functional network connectivity and task-modulation: application to schizophrenia. Magnetic Resonance Materials in Physics, Biology and Medicine, 23(5), 351-366. doi:10.1007/s10334-010-0197-8

Saxe, G. N., Calderone, D., \& Morales, L. J. (2018). Brain entropy and human intelligence: A resting-state fMRI study. PloS One, 13(2), e0191582. doi:10.1371/journal.pone.0191582

Scarpetta, S., Apicella, I., Minati, L., \& de Candia, A. (2018). Hysteresis, neural avalanches, and critical behavior near a first-order transition of a spiking neural network. Physical Review E, 97(6), 062305. doi:10.1103/PhysRevE.97.062305

Scott, G., Fagerholm, E. D., Mutoh, H., Leech, R., Sharp, D. J., Shew, W. L., \& Knöpfel, T. (2014). Voltage Imaging of Waking Mouse Cortex Reveals Emergence of Critical Neuronal Dynamics. The Journal of Neuroscience, 34(50), 16611. doi:10.1523/JNEUROSCI.3474-14.2014

Sethna, J. P., Dahmen, K. A., \& Myers, C. R. (2001). Crackling noise. Nature, 410(6825), 242-250. doi:10.1038/35065675

Shew, W. L., \& Plenz, D. (2012). The Functional Benefits of Criticality in the Cortex. The Neuroscientist, 19(1), 88-100. doi:10.1177/1073858412445487

Shew, W. L., Yang, H., Petermann, T., Roy, R., \& Plenz, D. (2009). Neuronal Avalanches Imply Maximum Dynamic Range in Cortical Networks at Criticality. The Journal of Neuroscience, 29(49), 15595. doi:10.1523/JNEUROSCI.3864-09.2009

Shew, W. L., Yang, H., Yu, S., Roy, R., \& Plenz, D. (2011). Information capacity and transmission are maximized in balanced cortical networks with neuronal avalanches. The 
Journal of neuroscience : the official journal of the Society for Neuroscience, 31(1), 55-63. doi:10.1523/JNEUROSCI.4637-10.2011

Shriki, O., Alstott, J., Carver, F., Holroyd, T., Henson, R. N. A., Smith, M. L., . . . Plenz, D. (2013). Neuronal avalanches in the resting MEG of the human brain. The Journal of neuroscience : the official journal of the Society for Neuroscience, 33(16), 7079-7090. doi:10.1523/JNEUROSCI.4286-12.2013

Smith, S. M., Nichols, T. E., Vidaurre, D., Winkler, A. M., Behrens, T. E. J., Glasser, M. F., . . Miller, K. L. (2015). A positive-negative mode of population covariation links brain connectivity, demographics and behavior. Nature Neuroscience, 18(11), 1565-1567. doi:10.1038/nn.4125

Smyser, C. D., Snyder, A. Z., Shimony, J. S., Mitra, A., Inder, T. E., \& Neil, J. J. (2016). Resting-State Network Complexity and Magnitude Are Reduced in Prematurely Born Infants. Cerebral cortex (New York, N.Y. : 1991), 26(1), 322-333. doi:10.1093/cercor/bhu251

Solovey, G., Miller, K., Ojemann, J., Magnasco, M., \& Cecchi, G. (2012). Self-Regulated Dynamical Criticality in Human ECoG. Frontiers in Integrative Neuroscience, 6, 44.

Song, B., Ma, N., Liu, G., Zhang, H., Yu, L., Liu, L., \& Zhang, J. (2019). Maximal flexibility in dynamic functional connectivity with critical dynamics revealed by fMRI data analysis and brain network modelling. Journal of Neural Engineering, 16(5), 056002. doi:10.1088/1741-2552/ab20bc

Sotiropoulos, S. N., Jbabdi, S., Xu, J., Andersson, J. L., Moeller, S., Auerbach, E. J., . . . Consortium, W. U.-M. H. (2013). Advances in diffusion MRI acquisition and processing in the Human Connectome Project. NeuroImage, 80, 125-143. doi:10.1016/j.neuroimage.2013.05.057

Sporns, O. (2013). Network attributes for segregation and integration in the human brain.
Current
Opinion
in Neurobiology,
23(2),
162-171. doi:https://doi.org/10.1016/j.conb.2012.11.015

Tagliazucchi, E., Balenzuela, P., Fraiman, D., \& Chialvo, D. (2012). Criticality in Large-Scale Brain fMRI Dynamics Unveiled by a Novel Point Process Analysis. Frontiers in Physiology, 3, 15.

Tagliazucchi, E., \& Chialvo, D. R. (2013). Brain complexity born out of criticality. AIP Conference Proceedings, 1510(1), 4-13. doi:10.1063/1.4776495

Tagliazucchi, E., Chialvo, D. R., Siniatchkin, M., Amico, E., Brichant, J.-F., Bonhomme, V., . . Laureys, S. (2016). Large-scale signatures of unconsciousness are consistent with a 
departure from critical dynamics. Journal of the Royal Society, Interface, 13(114), 20151027-20151027. doi:10.1098/rsif.2015.1027

Timme, N. M., Marshall, N. J., Bennett, N., Ripp, M., Lautzenhiser, E., \& Beggs, J. M. (2016). Criticality Maximizes Complexity in Neural Tissue. Frontiers in Physiology, 7, 425-425. doi:10.3389/fphys.2016.00425

Tononi, G., Edelman, G. M., \& Sporns, O. (1998). Complexity and coherency: integrating information in the brain. Trends in Cognitive Sciences, 2(12), 474-484. doi:10.1016/S1364-6613(98)01259-5

Tononi, G., Sporns, O., \& Edelman, G. M. (1994). A measure for brain complexity: relating functional segregation and integration in the nervous system. Proceedings of the National Academy of Sciences, 91(11), 5033. doi:10.1073/pnas.91.11.5033

Touboul, J., \& Destexhe, A. (2010). Can power-law scaling and neuronal avalanches arise from stochastic dynamics? PloS One, 5(2), e8982-e8982. doi:10.1371/journal.pone.0008982

Van Essen, D. C., Smith, S. M., Barch, D. M., Behrens, T. E. J., Yacoub, E., Ugurbil, K., \& Consortium, W. U.-M. H. (2013). The WU-Minn Human Connectome Project: an overview. NeuroImage, 80, 62-79. doi:10.1016/j.neuroimage.2013.05.041

Vijayraghavan, S., Wang, M., Birnbaum, S. G., Williams, G. V., \& Arnsten, A. F. T. (2007). Inverted-U dopamine D1 receptor actions on prefrontal neurons engaged in working memory. Nature Neuroscience, 10(3), 376-384. doi:10.1038/nn1846

Wang, B., Niu, Y., Miao, L., Cao, R., Yan, P., Guo, H., . . . Zhang, H. (2017). Decreased Complexity in Alzheimer's Disease: Resting-State fMRI Evidence of Brain Entropy Mapping. Frontiers in Aging Neuroscience, 9, 378-378. doi:10.3389/fnagi.2017.00378

Wang, R., Lin, P., Liu, M., Wu, Y., Zhou, T., \& Zhou, C. (2019). Hierarchical Connectome Modes and Critical State Jointly Maximize Human Brain Functional Diversity. Physical Review Letters, 123(3), 038301. doi:10.1103/PhysRevLett.123.038301

Wang, R., Liu, M., Cheng, X., Wu, Y., Hildebrandt, A., \& Zhou, C. (2021). Segregation, integration, and balance of large-scale resting brain networks configure different cognitive abilities. Proceedings of the National Academy of Sciences, 118(23), e2022288118. doi:10.1073/pnas.2022288118

Wendelken, C., Nakhabenko, D., Donohue, S. E., Carter, C. S., \& Bunge, S. A. (2007). "Brain Is to Thought as Stomach Is to ??": Investigating the Role of Rostrolateral Prefrontal Cortex in Relational Reasoning. Journal of Cognitive Neuroscience, 20(4), 682-693. doi:10.1162/jocn.2008.20055 
1277

1278

1279

1280

1281

1282

1283

1284

1285

1286

1287

1288

1289

1290

1291

1292

1293

1294

1295

1296

1297

1298

1299

1300

1301

1302

1303

1304

1305

1306

Xia, M., Wang, J., \& He, Y. (2013). BrainNet Viewer: A Network Visualization Tool for Human Brain Connectomics. PloS One, 8(7), e68910. doi:10.1371/journal.pone.0068910

Yang, H., Shew, W. L., Roy, R., \& Plenz, D. (2012). Maximal Variability of Phase Synchrony in Cortical Networks with Neuronal Avalanches. The Journal of Neuroscience, 32(3), 1061. doi:10.1523/JNEUROSCI.2771-11.2012

Yao, Y., Lu, W. L., Xu, B., Li, C. B., Lin, C. P., Waxman, D., \& Feng, J. F. (2013). The Increase of the Functional Entropy of the Human Brain with Age. Scientific Reports, 3(1), 2853. doi:10.1038/srep02853

Yeh, F.-C., Liu, L., Hitchens, T. K., \& Wu, Y. L. (2017). Mapping immune cell infiltration using restricted diffusion MRI. Magnetic Resonance in Medicine, 77(2), 603-612. doi:10.1002/mrm.26143

Yeh, F.-C., \& Tseng, W.-Y. I. (2011). NTU-90: A high angular resolution brain atlas constructed by q-space diffeomorphic reconstruction. NeuroImage, 58(1), 91-99. doi:https://doi.org/10.1016/j.neuroimage.2011.06.021

Yeh, F.-C., Verstynen, T. D., Wang, Y., Fernández-Miranda, J. C., \& Tseng, W.-Y. I. (2013). Deterministic Diffusion Fiber Tracking Improved by Quantitative Anisotropy. PloS One, 8(11), e80713. doi:10.1371/journal.pone.0080713

Yeh, F., Wedeen, V. J., \& Tseng, W. I. (2010). Generalized q-Sampling Imaging. IEEE Transactions on Medical Imaging, 29(9), 1626-1635. doi:10.1109/TMI.2010.2045126

Zahrt, J., Taylor, J. R., Mathew, R. G., \& Arnsten, A. F. (1997). Supranormal stimulation of D1 dopamine receptors in the rodent prefrontal cortex impairs spatial working memory performance. The Journal of neuroscience : the official journal of the Society for Neuroscience, 17(21), 8528-8535. doi:10.1523/JNEUROSCI.17-21-08528.1997

Zalesky, A., Fornito, A., Harding, I. H., Cocchi, L., Yücel, M., Pantelis, C., \& Bullmore, E. T. (2010). Whole-brain anatomical networks: Does the choice of nodes matter? NeuroImage, 50(3), 970-983. doi:https://doi.org/10.1016/j.neuroimage.2009.12.027

Zapperi, S., Lauritsen, K. B., \& Stanley, H. E. (1995). Self-Organized Branching Processes: Mean-Field Theory for Avalanches. Physical Review Letters, 75(22), 4071-4074. doi:10.1103/PhysRevLett.75.4071 
Table 1. The brain regions exhibited significant correlation between SE and fluid intelligence.

\begin{tabular}{|c|c|c|c|c|c|c|}
\hline Lobe & Gyrus & Left/Right & $\begin{array}{c}\text { Brodmann } \\
\text { area }\end{array}$ & $\begin{array}{c}\text { MNI } \\
\text { coordinate }\end{array}$ & $\mathrm{R}$ value & FDR \\
\hline Frontal & Superior Frontal & SFG_L_7_3 & $8,9,10$ & {$[-11,49,40]$} & 0.2058 & 0.0120 \\
\hline \multirow[t]{12}{*}{ Lobe } & Gyrus (SFG) & SFG_R_7_3 & $8,9,10$ & {$[13,48,40]$} & 0.1795 & 0.0264 \\
\hline & & SFG_R_7_7 & 10 & {$[8,58,13]$} & 0.1784 & 0.0268 \\
\hline & Middle Frontal & MFG_L_7_1 & 9,46 & {$[-27,43,31]$} & 0.1827 & 0.0264 \\
\hline & Gyrus (MFG) & MFG_L_7_3 & $10,11,46$ & {$[-28,56,12]$} & 0.1947 & 0.0176 \\
\hline & & MFG_R_7_3 & $9,10,46$ & {$[28,55,17]$} & 0.2706 & 0.0007 \\
\hline & & MFG_L_7_5 & $8,9,44,46$ & {$[-33,23,45]$} & 0.1871 & 0.0229 \\
\hline & & MFG_R_7_5 & $9,44,45,46$ & {$[42,27,39]$} & 0.2522 & 0.0017 \\
\hline & & MFG_L_7_7 & $10,11,47$ & {$[-26,60,-6]$} & 0.1662 & 0.0429 \\
\hline & $\begin{array}{l}\text { Inferior Frontal } \\
\text { Gyrus (IFG) }\end{array}$ & IFG_R_6_5 & 45,47 & {$[42,22,3]$} & 0.1820 & 0.0264 \\
\hline & Precentral Gyrus & PrG_L_6_1 & 4,6 & {$[-49,-8,39]$} & 0.1797 & 0.0264 \\
\hline & $(\mathrm{PrG})$ & PrG_R_6_1 & 4,6 & {$[55,-2,33]$} & 0.1718 & 0.0344 \\
\hline & $\begin{array}{c}\text { Paracentral Lobule } \\
\text { (PCL) }\end{array}$ & PCL_R_2_2 & 4,6 & {$[5,-21,61]$} & 0.1728 & 0.0344 \\
\hline $\begin{array}{l}\text { Tempora } \\
1 \text { Lobe }\end{array}$ & $\begin{array}{l}\text { Inferior Temporal } \\
\text { Gyrus (ITG) }\end{array}$ & ITG_R_7_5 & $20,21,37$ & {$[54,-57,-8]$} & 0.2066 & 0.0120 \\
\hline \multirow[t]{10}{*}{$\begin{array}{l}\text { Parietal } \\
\text { Lobe }\end{array}$} & $\begin{array}{c}\text { Superior Parietal } \\
\text { Lobule (SPL) }\end{array}$ & SPL_R_5_5 & 7,40 & {$[31,-54,53]$} & 0.1717 & 0.0344 \\
\hline & Angular Gyrus & IPL_R_6_1 & 39 & {$[45,-71,20]$} & 0.1687 & 0.0390 \\
\hline & $(\mathrm{AG})$ & IPL_R_6_2 & 7,39 & {$[39,-65,44]$} & 0.2373 & 0.0028 \\
\hline & & IPL_L_6_5 & 39 & {$[-47,-65,26]$} & 0.2011 & 0.0140 \\
\hline & & IPL_R_6_5 & 39 & {$[53,-54,25]$} & 0.2284 & 0.0043 \\
\hline & Supramarginal & IPL_L_6_3 & $2,3,40$ & {$[-51,-33,42]$} & 0.1997 & 0.0140 \\
\hline & Gyrus (SG) & IPL_R_6_3 & $2,3,40$ & {$[47,-35,45]$} & 0.2061 & 0.0120 \\
\hline & & IPL_R_6_4 & 2,40 & {$[57,-44,38]$} & 0.2369 & 0.0028 \\
\hline & Precuneus (Pcun) & Pcun_L_4_4 & 7 & {$[-6,-55,34]$} & 0.1810 & 0.0264 \\
\hline & & Pcun_R_4_4 & 7 & {$[6,-54,35]$} & 0.1899 & 0.0219 \\
\hline $\begin{array}{l}\text { Limbic } \\
\text { Lobe }\end{array}$ & $\begin{array}{l}\text { Cingulate Gyrus } \\
\text { (CG) }\end{array}$ & CG_L_7_6 & 23 & {$[-7,-23,41]$} & 0.1868 & 0.0229 \\
\hline $\begin{array}{l}\text { Occipital } \\
\text { Lobe }\end{array}$ & $\begin{array}{l}\text { Superior Occipital } \\
\text { Gyrus (sOcG) }\end{array}$ & sOcG_R_2_1 & 18,19 & {$[16,-85,34]$} & 0.2051 & 0.0120 \\
\hline
\end{tabular}


A
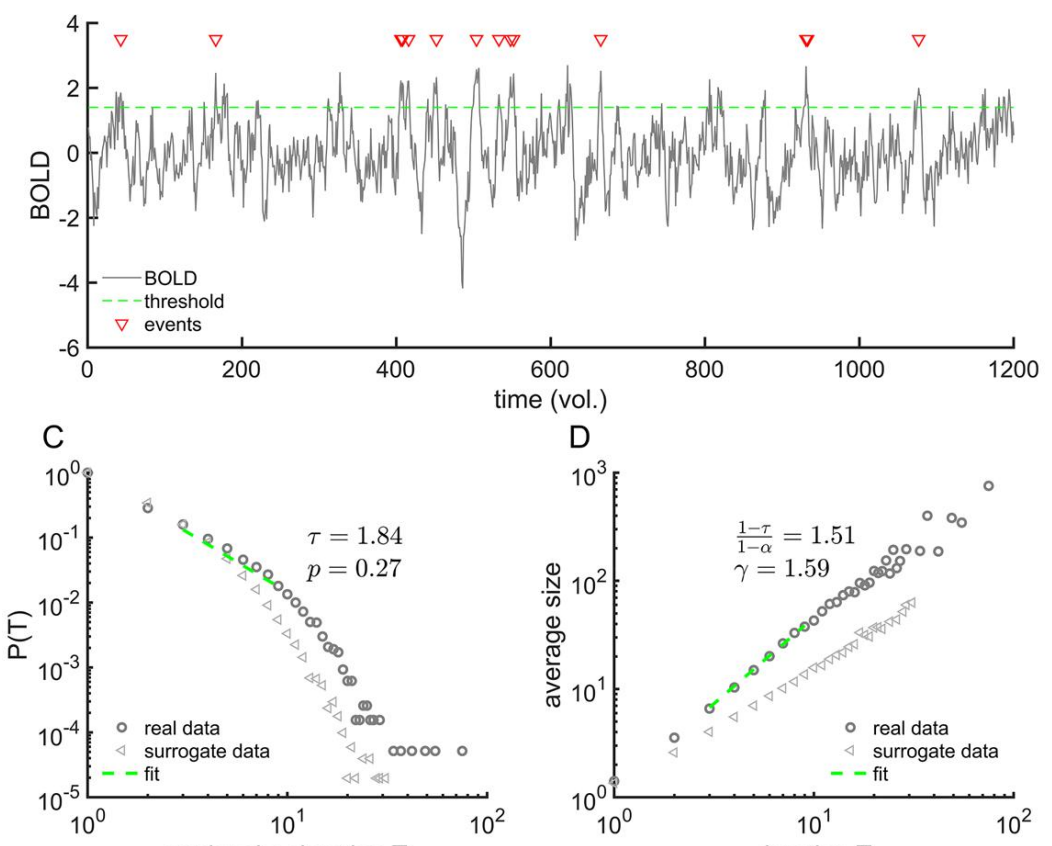

avalanche duration $\mathrm{T}$

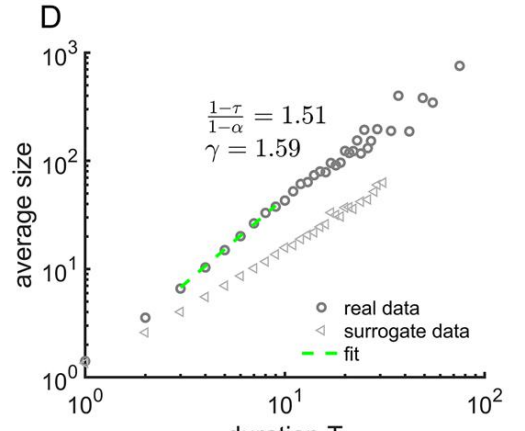

duration $\mathrm{T}$
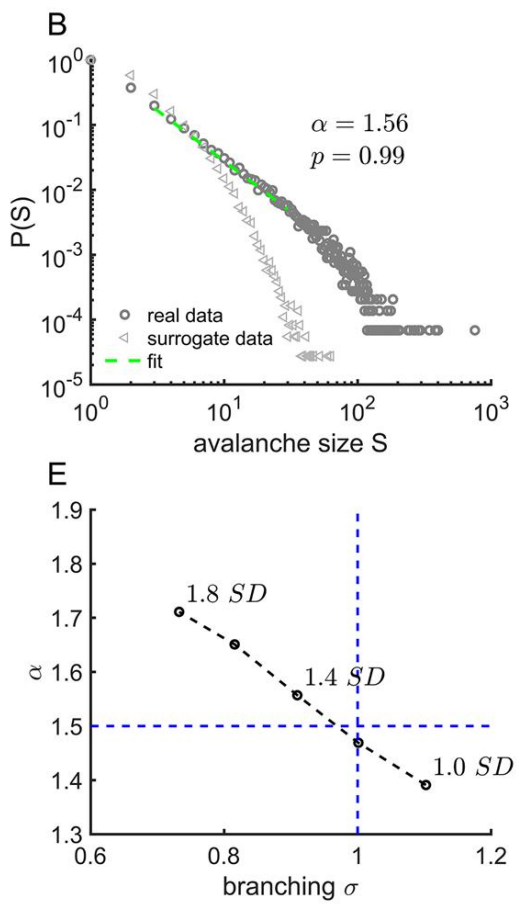

1312 Figure 1. Avalanche statistics obtained from group-level analysis. A. Example of a point

1313 process (red triangles) extracted from one normalized ROI BOLD signal. B. The probability

1314 distributions of group-aggregated avalanche sizes for the threshold $1.4 \mathrm{SD}$ and the time bin

1315 width of 1 volume (vol.) in the fMRI data. The distributions are well approximated by power

1316 law with an exponent of $\alpha=1.56$ with Clauset's test $p=0.99$, corresponding to $\kappa=$

13170.9912 (histogram bins $=40$ ) and branching parameter $\sigma=0.9097$. C. The distribution of

1318 avalanche durations can be fitted well by a power law with an exponent of $\tau=1.84$ under

1319 the condition described in $\mathbf{B}$, with Clauset's test $p=0.3$. D. There is a relation between the

1320 sizes and duration of the avalanches with a positive index $\gamma=1.59$, which is close to $\frac{1-\tau}{1-\alpha}=$

1321 1.51. In B-D, the grey open triangles were calculated from the surrogate data. E. The

1322 branching ratio and power-law scaling exponents $\alpha$ of avalanche sizes for different thresholds used to define the point process. 

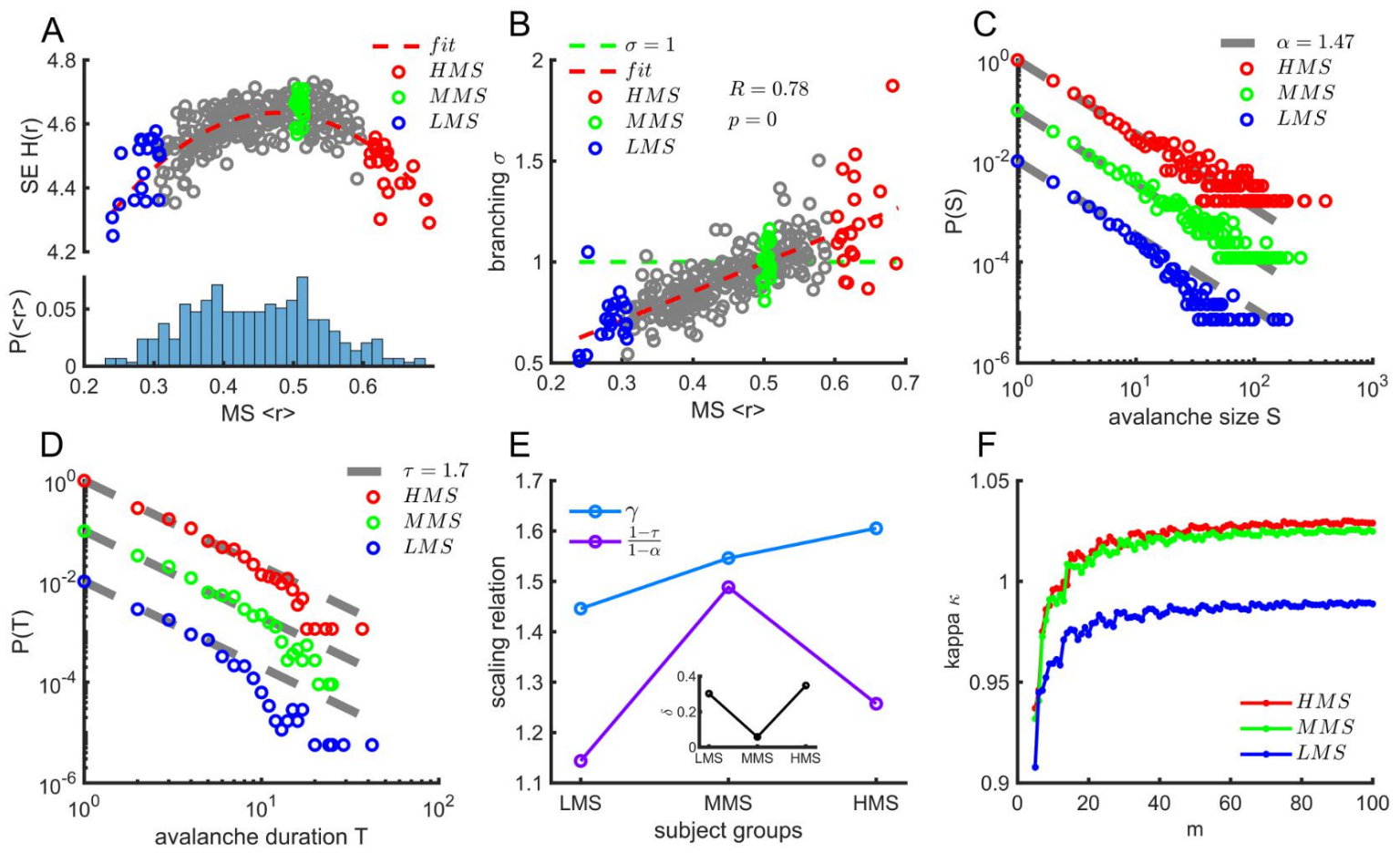

1327 Top panel: The inverted-U trajectory of the MS $\langle r\rangle$ vs. SE $H(r)$. The red dash line 1328 represents the quadratic fit of the data $\left(F=188.758, p<0.001\right.$, adjusted $\left.R^{2}=0.561\right)$. Bottom panel: The frequency count for the distribution of MS $\langle r\rangle$. B. The branching parameters $\sigma$ vs. $\langle r\rangle$ for each subject. The green dashed line indicates $\sigma=1$. The Pearson correlation value $R$ and the $p$ value are shown in the figure. The red dashed line represents the linear 1332 regression. For further analysis, we selected three representative groups of subjects according 1333 to their synchronization level: namely, LMS group $(\langle r\rangle=0.2824 \pm 0.0219$, blue open 1334 circles in A and B), MMS group $(\langle r\rangle=0.5041 \pm 0.0042$, green open circles in A and B) and HMS group $(\langle r\rangle=0.6304 \pm 0.0246$, red open circles in A and B). C. Avalanche size 1336 distributions for the LMS group, MMS group, and HMS group. To show the difference 1337 between these groups, we used gray lines with $\alpha=1.47$ to guide the eyes. The 
1340 Avalanche duration distributions for three groups in C. To show the difference between these

1341 groups, we used gray lines with $\tau=1.7$ to guide the eyes. E. Scaling relations for the three

1342 groups. The blue line and purple line correspond to $\gamma$ and $\frac{\tau-1}{\alpha-1}$, respectively. In the inset, $1343 \delta=\left|\gamma-\frac{\tau-1}{\alpha-1}\right|$ indicates the distance to the critical point. F. The dependence of $\kappa$ on the 1344 numbers $m$ of histogram bins for the three groups. 

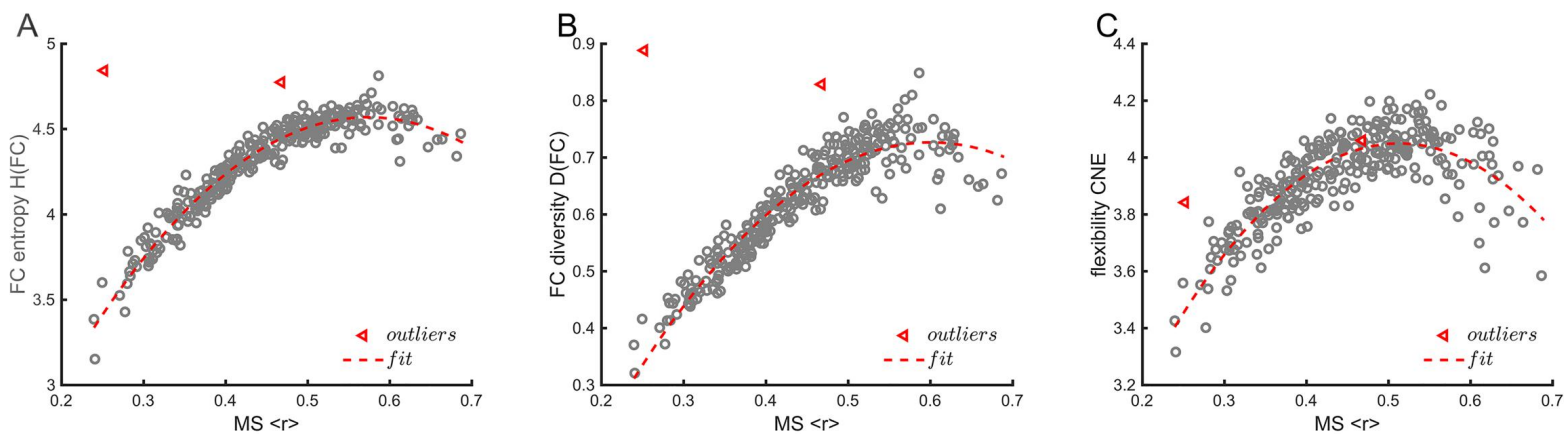

1357 Figure 3. Dependence of complexity in the FC network on the MS of BOLD signals. A.

1358 FC entropy $H(F C)$ as a function of MS $\langle r\rangle$. Red dashed line: quadratic fitting $(F=$

1359 2287.892, $p<0.001$, adjusted $\left.R^{2}=0.940\right)$. B. FC diversity $D(F C)$ as a function of MS $\langle r\rangle$.

1360 Red dashed line: quadratic fitting $\left(F=1226.057, p<0.001\right.$, adjusted $\left.R^{2}=0.894\right)$. C. FC

1361 flexibility $C N E$ as a function of MS $\langle r\rangle$. Red dashed line: quadratic fitting $(F=366.851$,

$1362 p<0.001$, adjusted $\left.R^{2}=0.715\right)$. The red open triangles represent participants with outliers in

1363 the quadratic fittings in A-B. 
A

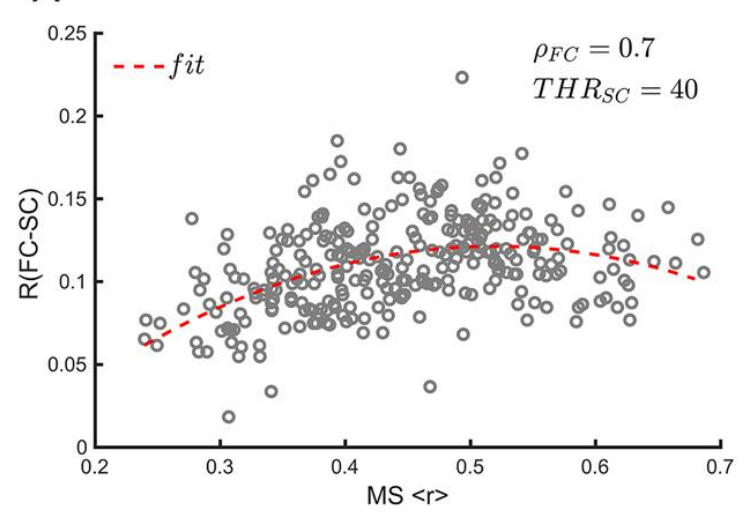

C

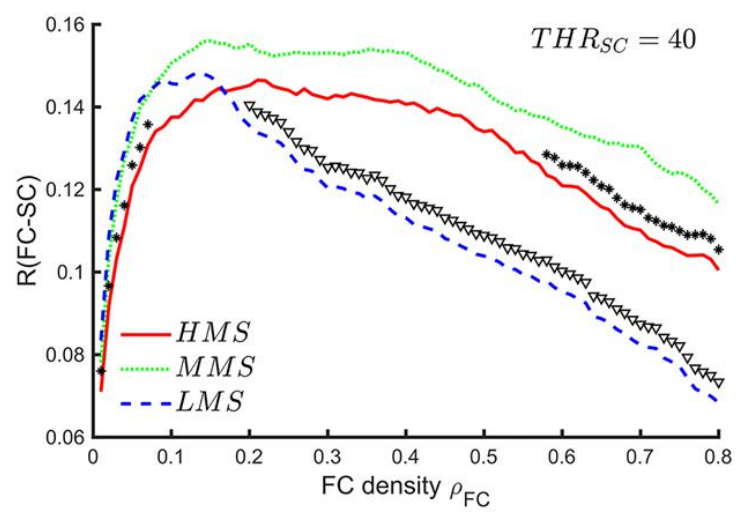

B

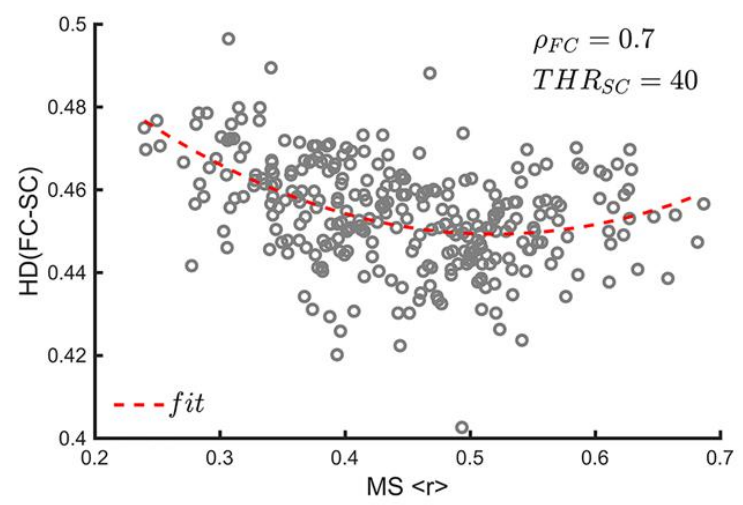

$\mathrm{D}$

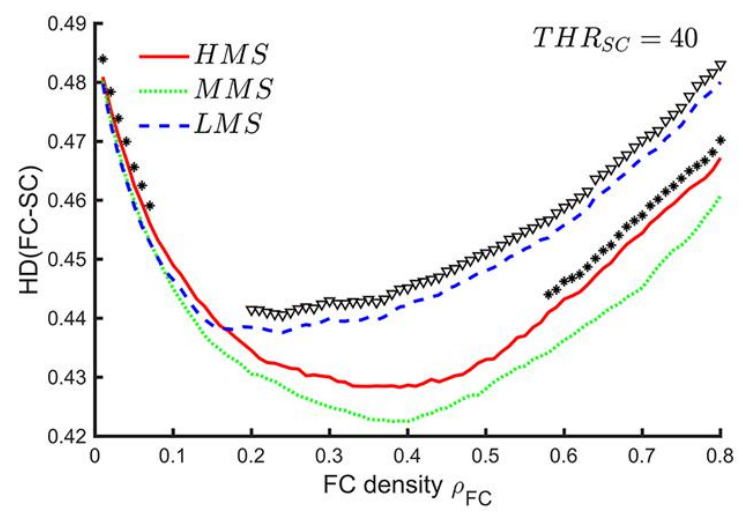

A. Pearson correlation between anatomical and functional networks as a function of MS $\langle r\rangle$. network $T H R_{S C}=40$ (corresponding to $\rho_{S C}=0.4836$ ) are shown in the figure. Red dashed

1369 line: quadratic fitting $\left(F=36.997, p<0.001\right.$, adjusted $\left.R^{2}=0.197\right)$, which is better than

1370 linear fitting ( $F=39.346, p<0.001$, adjusted $R^{2}=0.115$ ). B. Hamming distance $H D(F C-S C)$ between anatomical and functional networks as a function of MS $\langle r\rangle$. Red

1372 dashed line: quadratic fitting $\left(F=36.997, p<0.001\right.$, adjusted $\left.R^{2}=0.197\right)$, which is better 1373 than linear fitting $\left(F=39.346, p<0.001\right.$, adjusted $\left.R^{2}=0.115\right)$. C. The Pearson correlation between anatomical and functional networks as a function of FC density $\rho_{F C} \quad\left(T H R_{S C}=40\right.$

1375 corresponding to $\rho_{S C}=0.4826$ ) for the HMS, MMS, and LMS groups. D. The Hamming 
1376 distance between anatomical and functional networks as a function of FC density $\rho_{F C}$

$1377\left(T H R_{S C}=40\right.$ corresponding to $\left.\rho_{S C}=0.4826\right)$ for the HMS, MMS, and LMS groups. In

1378 C-D, the stars indicate significant differences between the HMS and MMS groups (two-tails

1379 two-sample t-test, $p<0.05$, uncorrected); the open triangles indicate significant differences

1380 between the LMS and MMS groups (two-tails two-sample t-test, $p<0.05$, uncorrected). 
A

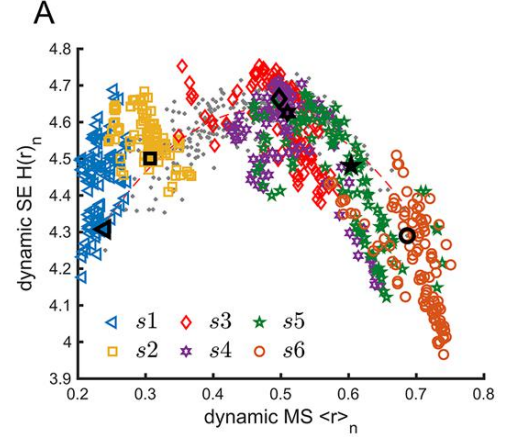

D

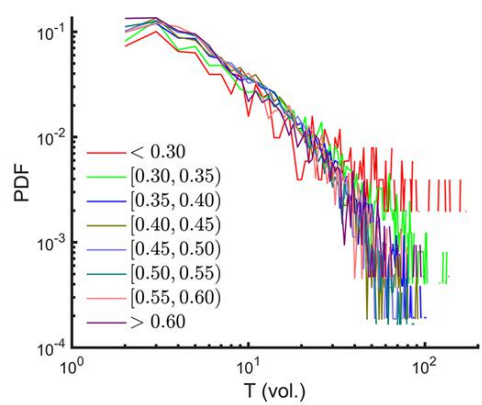

B
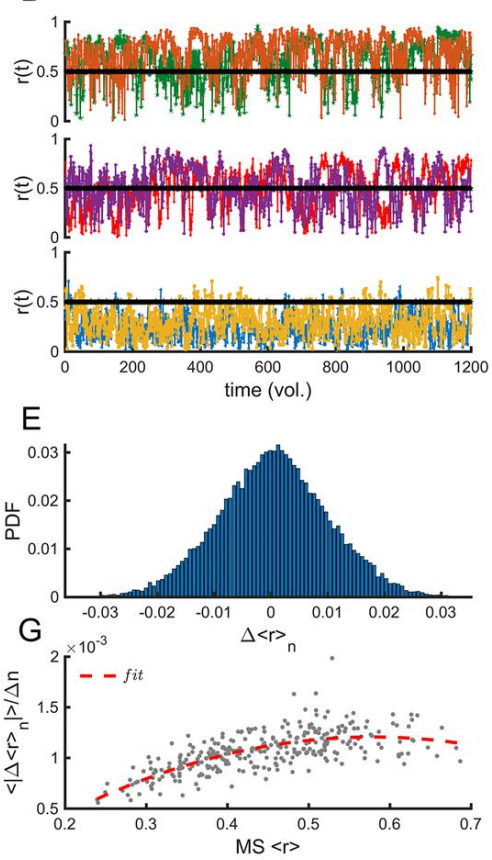

C
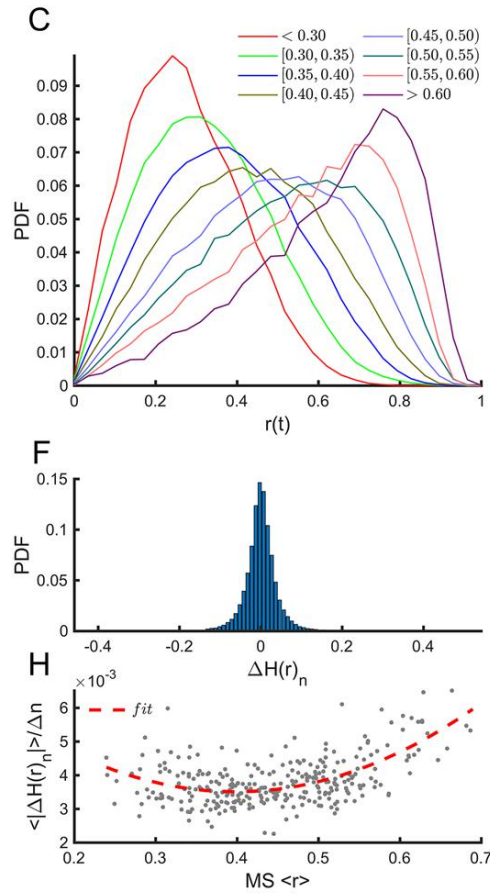

Figure 5. The dynamic phase transition in individual subjects' brains. A. The dependence

1384 of dynamic SE $H(r)_{n}$ on dynamic MS $\langle r\rangle_{n}$ from six subjects selected randomly from the

Kuramoto order parameter $r(t)$ for six subjects as demonstrated in figure a (with the same

color). C. The normalized frequency count of $r(t)$ for different levels of $\langle r\rangle$, indicated by lines with different colors. D. The dwell time (the time interval between two successive critical point crossing events) distribution for different levels of $\langle r\rangle$. (E, F). The distribution of vertical and horizontal moving distances of phase points in one step of the sliding window.

1392 (G, H). The vertical and horizontal velocities of state points of each subject as a function of their MS $\langle r\rangle$. The vertical and horizontal velocities were calculated by $\frac{\left\langle\left|\Delta\langle r\rangle_{n}\right|\right\rangle}{\Delta n}$ and $\frac{\left\langle\left|\Delta\langle r\rangle_{n}\right|\right\rangle}{\Delta n}$, where the symbol $|\cdot|$ indicates the absolute value, and $\langle\cdot\rangle$ was the average across all the windows. $\Delta n$ is the step used to slide the windows. Here, $\Delta \mathrm{n}=10$ time points (volumes). Red 
1396 dashed lines in G-H: quadratic fitting $\left(F=139.316, p<0.001\right.$, adjusted $R^{2}=0.485$ in

$1397(\mathrm{G}) ; F=81.181, p<0.001$, adjusted $R^{2}=0.353$ in figure $\left.\mathrm{h}\right)$. Both quadratic fittings were

1398 better than linear fitting (adjusted $R^{2}=0.407$ in $\mathbf{G}$ and adjusted $R^{2}=0.173$ in $\mathbf{H}$ ).

1399

1400

1401

1402

1403 
A

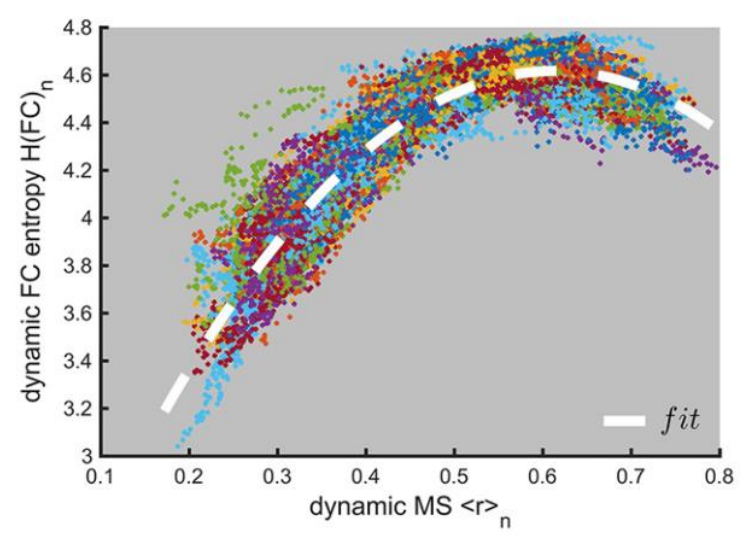

C

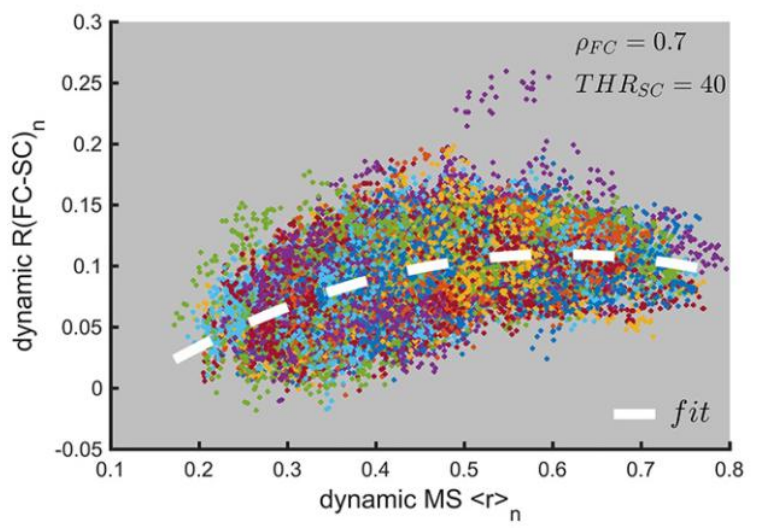

B

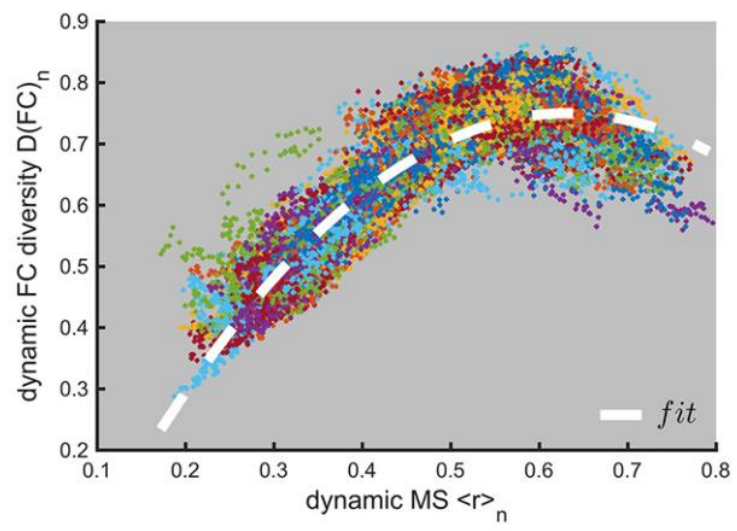

$\mathrm{D}$

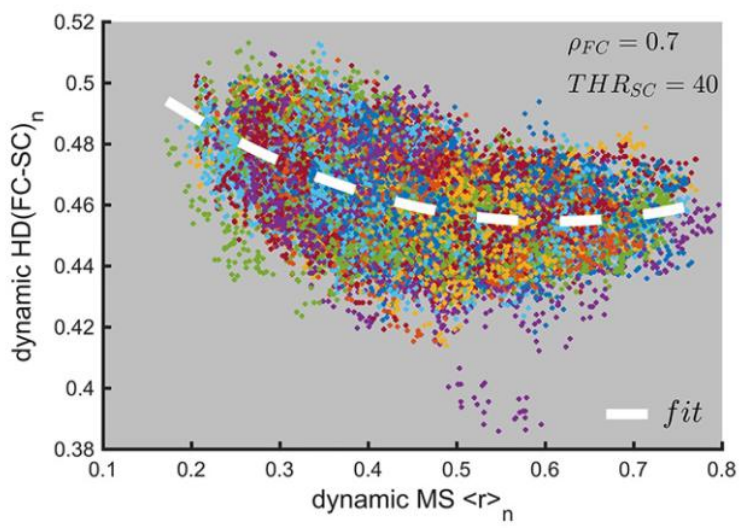

1406 during the phase transition of brains. A. The dependence of dynamic FC entropy as a

1407 function of instantaneous MS; thick dashed white line: quadratic fitting $(F=106350.82$,

$1408 p<0.001$, adjusted $\left.R^{2}=0.877\right)$. B. The dependence of dynamic FC diversity as a

1409 function of instantaneous MS. Thick dashed white line: quadratic fitting $(F=80261.492$,

$1410 p<0.001$, adjusted $\left.\mathrm{R}^{2}=0.843\right)$. C. The dependence of dynamic FC-SC correlation as a

1411 function of instantaneous MS; thick dashed white line: quadratic fitting $(F=5519.072$,

$1412 p<0.001$, adjusted $R^{2}=0.270$ ), which is better than linear fitting (adjusted $R^{2}=0.225$ ).

1413 D. The dependence of dynamic FC-SC Hamming distance as a function of instantaneous MS;

1414 thick dashed white line: quadratic fitting $(F=5519.072, p<0.001$, adjusted 
$1415 R^{2}=0.270$ ), which is better than linear fitting (adjusted $R^{2}=0.225$ ). In A-D, each dot

1416 represents a calculation from one window. The dots with the same color represent the

1417 calculation for one subject. However, due to the limited number of colors used, different

1418 subjects may share the same color. In C-D, a link density of 0.7 was used to obtain the binary

1419 FC network, and a threshold of 40 was used to obtain the group-aggregated structural

1420 network.

1421

1422 
A

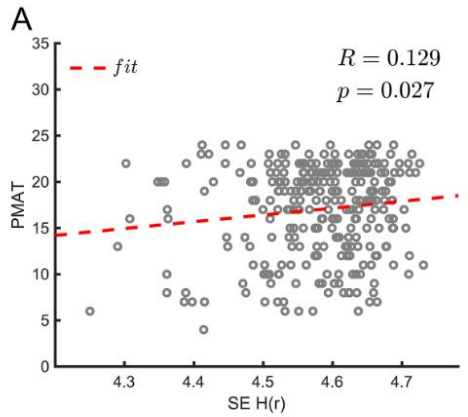

$\mathrm{D}$

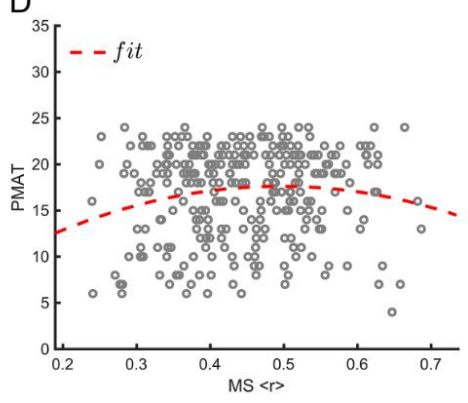

B
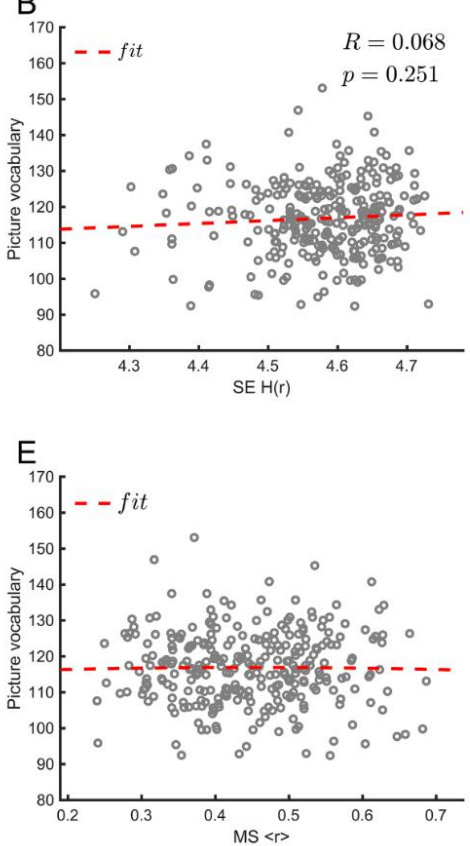

C

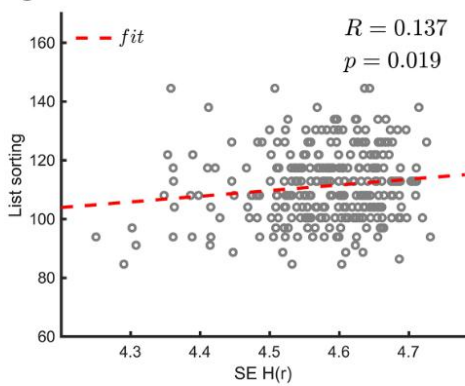

$\mathrm{F}$

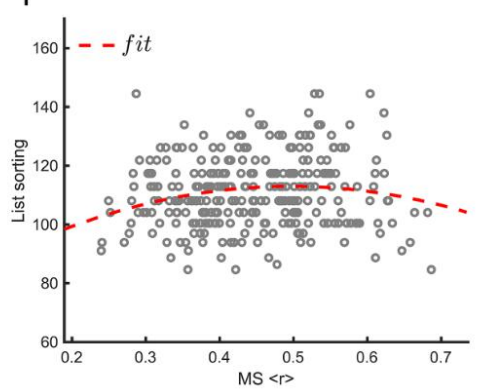

1424 Figure 7. Correlations between cognitive performance scores and SE, as well as MS.

1425 A-C. Correlation between SE and PMAT scores, picture vocabulary test scores, as well as the

1426 list sorting working memory test scores. Red dashed lines in A-C: linear fitting. D.

1427 Scatterplot of the PMAT scores against the MS. The red dashed line represents the significant 1428 quadratic fit of the data $\left(F=3.145, p=0.045\right.$, adjusted $\left.R^{2}=0.015\right)$, which is better than

1429 the linear fitting (adjusted $R^{2}=0.004$ ). E. Scatterplot of the picture vocabulary test scores 1430 against the MS. Both the linear and quadratic regressions are not significant (linear: $p=$ 14310.991 ; quadratic: $p=0.988)$. F. Scatterplot of the list sorting working memory test scores against the MS. The red dashed line represents the significant quadratic fit of the data $(F=$ 1433 4.376, $p=0.013$, adjusted $R^{2}=0.023$ ), which is better than linear fitting (adjusted $R^{2}=$ 0.008 ). 
$\mathbf{L}$
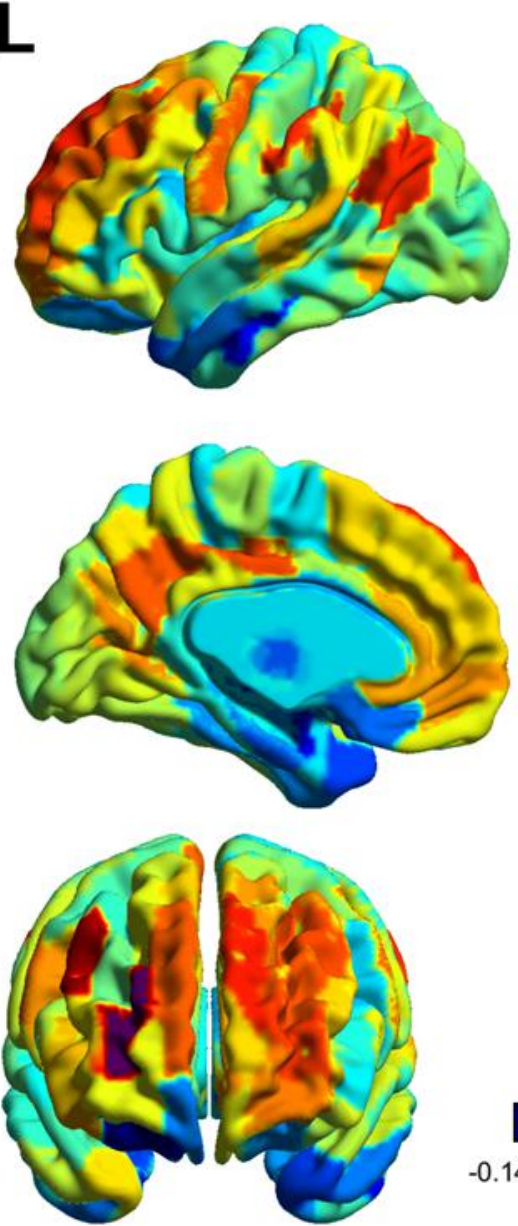
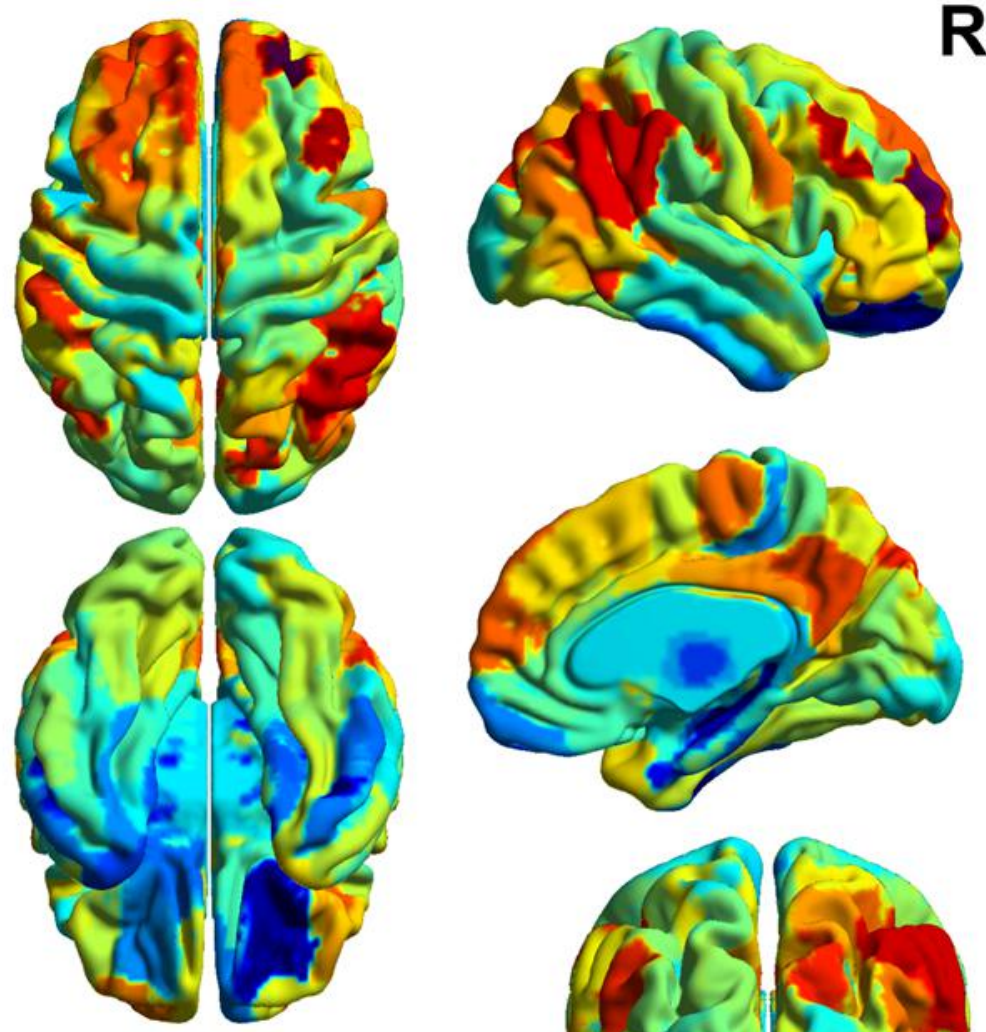

$-0.1484$

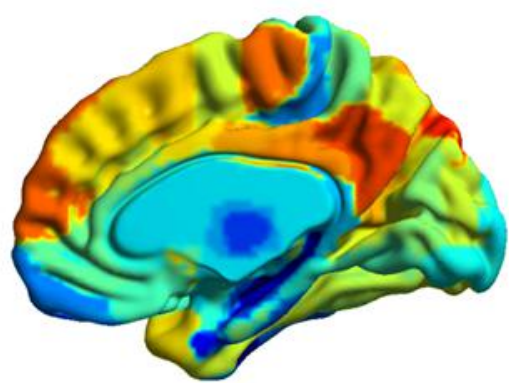

0.2706

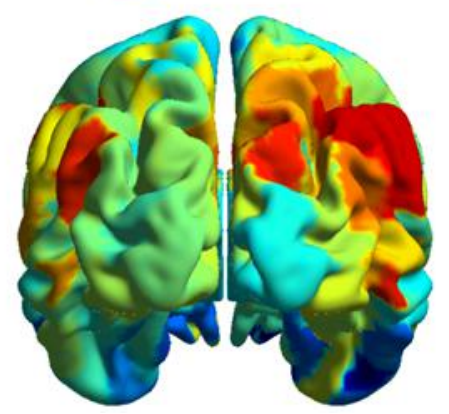

1436 Figure 8. The brain map for correlations between regional SE and fluid intelligence. The

1437 color bar indicated the Pearson correlation value between regional SE and PMAT. The

1438 cortical and subcortical regions were defined by the Human Brainnetome Atlas

1439 (http://atlas.brainnetome.org/bnatlas.html). Data was visualized using BrainNet Viewer (Xia,

1440 Wang, \& He, 2013). 